\title{
Molecular catalysts for artificial photosynthesis: general discussion
}

\author{
Mei Wang, Vincent Artero, Leif Hammarström, Jose Martinez, \\ Joshua Karlsson, Devens Gust, Peter Summers, Charles Machan, \\ Peter Brueggeller, Christopher D. Windle, Yosuke Kageshima, \\ Richard Cogdell, Kristine Rodulfo Tolod, Alexander Kibler, \\ Dogukan Hazar Apaydin, Etsuko Fujita, Johannes Ehrmaier, \\ Seigo Shima, Elizabeth Gibson, Ferdi Karadas, Anthony Harriman, \\ Haruo Inoue, Akihiko Kudo, Tomoaki Takayama, Michael Wasielewski, \\ Flavia Cassiola, Masayuki Yagi, Hitoshi Ishida, Federico Franco, \\ Sang Ook Kang, Daniel Nocera, Can Li, Fabio Di Fonzo, \\ Hyunwoong Park, Licheng Sun, Tohru Setoyama, Young Soo Kang, \\ Osamu Ishitani, Jian-Ren Shen, Ho-Jin Son and Shigeyuki Masaoka
}

Leif Hammarström opened discussion of the paper by Licheng Sun: You indicated using separate panels for PV and solar heating on the same roof top which compete for space in the sun. Instead, have you considered using the heat that is generated from the PV and circulating the water to the electrolyzer? Then you also increase the efficiency of the PV.

Licheng Sun responded: That is an even better idea, and solves the problem of competing roof area.

Masayuki Yagi asked: I am interested in the temperature dependence of the water oxidation catalysis. The linear relationship between the current density and temperature shows that the enhanced water oxidation is not ascribed to an activation process. If it involves the usual activation process, there is a linear relationship between the logarithm of the current density and the inverse of the temperature. How could you explain the enhancement of the water oxidation catalysis?

Licheng Sun replied: In addition to the thermodynamic reasons, kinetics processes are the major contributors to these temperature effects.

Masayuki Yagi remarked: Diffusion of $\mathrm{H}^{+}$and proton acceptors (if needed) could be important for electrochemical water oxidation. Is the enhanced water oxidation catalysis temperature due to diffusion of $\mathrm{H}^{+}$and proton acceptors? Did 
you check the potential of the reference electrode when the temperature is changed?

Licheng Sun responded: Mass transport is a factor for this temperature effect.

Masayuki Yagi remarked: Is it possible to observe a similar temperature dependence of water oxidation catalysis under stirring conditions? Stirring conditions might exclude the influence of the diffusion of $\mathrm{H}^{+}$and proton acceptors.

Licheng Sun answered: All measurements performed under different temperatures are under stirring conditions.

Kristine Tolod asked: Does elevating the operating temperature have any effect on the surface of the catalyst? Also, can you please comment on the stability of the catalysts when they are used at these elevated temperatures of up to $65{ }^{\circ} \mathrm{C}$ ?

Licheng Sun answered: No obvious effect on the surface and stability of the catalyst by elevating the temperature to $65{ }^{\circ} \mathrm{C}$ was observed.

Kristine Tolod commented: Have you observed any notable degradation concerning the catalyst and its performance?

Licheng Sun answered: No catalyst degradation has been observed.

Akihiko Kudo asked: The temperature dependence of electrochemical reactions is very important. Efficiency and product selectivity of electrochemical $\mathrm{CO}_{2}$ reduction also depend significantly on temperature as I have reported. ${ }^{1}$

1 A. Kudo, S. Nakagawa, A. Tsuneto and T. Sakata, J. Electrochem. Soc., 1993, 140, 1541.

Licheng Sun answered: We have not studied the temperature effect of the $\mathrm{CO}_{2}$ reduction, your earlier work definitely broadened the knowledge on such an effect.

Akihiko Kudo commented: Are there any tendencies in the temperature dependence among different oxygen-evolving catalysts? What does it mean if the temperature dependence is similar? The result should suggest some factors regarding the kinetics and mechanisms such as the rate-determining step.

Licheng Sun replied: We have studied Co oxides, Ni oxides and $\mathrm{Cu}$ oxides. They all showed such a temperature effect on the performance. These results suggest the kinetic as well as thermodynamic effect.

Vincent Artero commented: To continue discussion of the concern raised by Professor Yagi, I think it is important to check if the kinetics follows the Eyring law. To do that you should first correct the potential vs. RHE from temperature effect using the Nernst law and then plot current versus temperature. If Eyring law is not followed, then maybe the mechanism changes and I suggest checking the 
Faradic yield to see if the system transitions from $\mathrm{O}_{2}$ formation to $\mathrm{H}_{2} \mathrm{O}_{2}$ formation.

Licheng Sun responded: Good suggestions. We will check if the kinetics follow the Eyring law, and the possible change of reaction mechanism to water oxidation.

Hyunwoong Park said: Experience has shown that there is an enhancement of electrochemical conductivity. In an impedance study, as the temperature increases, the conductivity increases. The conductivity should be low. Try the same experiment with thicker films. You may have different results with pure metals.

Licheng Sun replied: Thank you for your suggestion, we may consider the use of thicker films.

Michael Wasielewski commented: You are certainly correct in pointing out that electrochemical processes are temperature dependent. The performance of photovoltaic cells is also temperature dependent. Thus, heating the overall system during solar illumination can be advantageous if the system is optimized to work at elevated temperatures. Have you considered the temperature dependence of the PV performance in your estimates of overall system efficiency? Would the PV temperature dependence help or hinder performance and are we correct in assuming that you are using silicon PV cells as your benchmark?

Licheng Sun responded: In this work, we did not consider the temperature effect of PV. In practice, it is even a better idea to use the heat generated by the PV for circulating the electrolyzer.

Devens Gust commented: With regard to the fact that silicon solar cells become less efficient as the temperature increases, I have heard that systems have been designed wherein water is circulated below and around the solar cell to remove excess heat. The hot water may then be used for domestic purposes, much as conventional solar hot water heaters are employed. I believe that some of these systems are commercially available, or nearly so.

Licheng Sun replied: The hot circulating water generated from the Si PV can definitely be used to heat the electrolyzer.

Fabio Di Fonzo asked: Have you considered in your model that placing your solar thermal panel next to a PV panel means doubling the solar power input? This is critical for a correct evaluation of the efficiency figure. A more correct architecture would be placing the thermal collector below, with respect the direction of the sun, the PV panel in order to fully exploit the solar spectrum. Would this work for your system?

Licheng Sun responded: Yes, we are fully aware that placing a solar thermal panel takes extra space. We are fully agree that a more correct architecture is needed, such as pacing the thermal collector below the PV panel. 
Ferdi Karadas opened discussion of the paper by Shigeyuki Masaoka: The paper clearly states that the iron complex is not stable at low and high pH media. Could you comment on the stability of the iron complex at high potentials? Do you believe it retains its structure or decomposes while it is oxidized to its +4 oxidation state?

Shigeyuki Masaoka responded: In the electrochemical measurements of the iron complex (Fig. 3 in the paper), the wave that corresponds to the oxidation of the $\mathrm{Fe}^{\mathrm{III}}$ species was not observed. The result indicates that the generation of high-valent $\mathrm{Fe}^{\mathrm{IV}}$ species is quite difficult in this case. This is may be because the localization of positive charge at a single iron center is not favorable. We recently found that the use of a multinuclear iron system affords the easily accessible $\mathrm{Fe}^{\mathrm{IV}}$ state due to its highly delocalized electronic structure. ${ }^{1}$

1 M. Okamura, M. Kondo, R. Kuga, Y. Kurashige, T. Yanai, S. Hayami, V. K. K. Praneeth, M. Yoshida, K. Yoneda, S. Kawata and S. Masaoka, Nature, 2016, 530, 465.

Ferdi Karadas said: The comparison of the Pourbaix diagrams indicates that the $\mathrm{Fe}^{2+/ 3+}$ redox occurs at much lower potentials compared to that of $\mathrm{Ru}^{2+/ 3+}$. However, there seems to be an opposite trend with the $3+/ 4+$ redox bands. While $\mathrm{Ru}^{3+/ 4+}$ redox is observed at the potentials applied $\mathrm{Fe}^{3+/ 4+}$ band is not observed at all. Could you comment on this? While the $3+$ oxidation state of $\mathrm{Fe}$ is easily accessible, the +4 oxidation state is not observed for iron. What could be the reason?

Shigeyuki Masaoka replied: Based on the results of the electrochemical measurements of the $\mathrm{Ru}-\mathrm{OH}_{2}$ complex, we believe that the oxidation of the $\mathrm{M}^{\mathrm{III}}$ species affords the $\mathrm{M}^{\mathrm{IV}}$-oxo species. When we consider the molecular orbitals of the $\mathrm{Fe}^{\mathrm{IV}}$-oxo and $\mathrm{Ru}^{\mathrm{IV}}$-oxo species, it is expected that the interaction between the $\mathrm{Ru}$ and oxygen atoms would be larger than that of the Fe and oxygen atoms due to the larger $4 \mathrm{~d}$ orbital of $\mathrm{Ru}$ compared to the $3 \mathrm{~d}$ orbital of Fe. Therefore, we currently think that this difference in the interaction between molecular orbitals affects the stability of $\mathrm{M}^{\mathrm{IV}}$-oxo species, which results in the shift of the oxidation potential. TD-DFT calculation of the $\mathrm{M}^{\mathrm{IV}}$-oxo species is currently ongoing to further clarify our idea.

Ferdi Karadas remarked: The study concludes with an interesting and important statement for iron complexes. The main problem with iron complexes is their tendency to degrade easily due to their high lability for substitution. Could you comment generally on the type of ligands that could be used to improve the stability of iron complexes?

Shigeyuki Masaoka replied: As you indicated, the main problem of the ironbased complex is its instability. In general, the utilization of multidentate ligands can prevent the liberation of metal ions during catalytic processes. However, in the case of iron complexes, this strategy cannot always work as indicated by the present study. As one of the solutions, the utilization of multinuclear complexes is effective in smoothly accumulating the positive charge and preventing the decomposition of the complex during the reaction. ${ }^{1}$ 
1 M. Okamura, M. Kondo, R. Kuga, Y. Kurashige, T. Yanai, S. Hayami, V. K. K. Praneeth, M. Yoshida, K. Yoneda, S. Kawata and S. Masaoka, Nature, 2016, 530, 465.

Leif Hammarström asked: If you look at your Pourbaix diagram, the first oxidation of the Fe complex is about $200 \mathrm{mV}$ less positive than that for Ru. It is likely higher oxidations are also lower. Is it possible you just do not have enough driving force to oxidize water with the Fe complex? Have you tried this at pH 5 to oxidise water, where the thermodynamics are more favorable? Your complex is also more stable at that $\mathrm{pH}$.

Shigeyuki Masaoka responded: As you indicated, the redox peak attributed to the $\mathrm{Fe}^{\mathrm{III}} / \mathrm{Fe}^{\mathrm{II}}$ redox couple was observed at a less positive potential than the $\mathrm{Ru}^{\mathrm{III}} /$ $\mathrm{Ru}^{\mathrm{II}}$ redox couple for $\mathrm{M}-\mathrm{OH}_{2}$ complexes. In contrast, the redox peak corresponding to the generation of the $\mathrm{Fe}^{\mathrm{IV}}$-oxo species, which can be a key intermediate to trigger the water oxidation reaction, was not observed in the current experimental condition. The result indicates that the oxidation of the $\mathrm{Fe}^{\mathrm{III}}$ species is extremely difficult, whereas the oxidation of the $\mathrm{Ru}^{\mathrm{III}}$ species easily proceeds within the potential window. We currently think that the difficulty in generating high-valent species is the main reason for the lower catalytic activity of the iron complex.

Vincent Artero remarked: Did you check if the wave seen in the CV results measured in propylene carbonate and in the presence of water corresponds to water oxidation?

Shigeyuki Masaoka responded: We have not checked if the irreversible wave observed in the presence of water corresponds to water oxidation yet. The controlled potential electrolysis (CPE) of the complexes will give quantitative information on the product of these catalytic reactions. We will perform the CPE experiments in near future and will report the results. Thank you for your kind suggestion.

Anthony Harriman remarked: Your $\mathrm{pH}$ dependent curves are analysed in what looks to be a rather simple manner. A straight line is drawn through what looks to be an inflection point. Maybe some useful information is lost by this approach. Would it be meaningful to analyse the $\mathrm{pH}$ profiles in more detail?

Shigeyuki Masaoka responded: Thank you for your suggestion. Before the submission of our manuscript, we also considered this point. According to your suggestion, we have also reanalyzed the electrochemical data considering several possibilities. As a result, we think that the analysis shown in Fig. 3 of the paper gave the most reasonable interpretation at this stage.

Anthony Harriman commented: It seems that ruthenium offers a better choice than iron at this stage. Is there any reason to persist with iron, other than cost and availability?

Shigeyuki Masaoka responded: This study indicated that the nature of iron ions are very different from that of ruthenium ions and a distinct strategy to construct the active catalyst is required. Our recent study clearly demonstrated 
that the iron catalyst based on appropriate design could serve as highly active and stable catalyst for water oxidation. ${ }^{1}$ Therefore, what we need to do is establish the appropriate design principle to obtain highly active and stable iron-based catalysts.

1 M. Okamura, M. Kondo, R. Kuga, Y. Kurashige, T. Yanai, S. Hayami, V. K. K. Praneeth, M. Yoshida, K. Yoneda, S. Kawata and S. Masaoka, Nature, 2016, 530, 465.

Can Li asked: Comparing the three different kinds of complex, have you ever tried to see the actual performance of the catalysts in the PC device? It might be very different, and be stable in the device despite not being stable here? It would be beneficial to have experimental results to show if these catalysts really work in a device.

Shigeyuki Masaoka answered: We have not checked the performance of the catalysts in a PC device yet. As you indicated, the catalytic activity of these catalysts can alter by the introduction into a PC device. We hope we can perform such investigations in future.

Seigo Shima opened discussion of the paper by Mei Wang: In your data indicating the kinetic parameters, you compared complex 1 and complex 2 . Why you did not use a compound for comparison which has an $\mathrm{NH}$ group instead of $\mathrm{CH}_{2}$ at the center of the bridge part because the complex 1 has a nitrogen connecting to the phenyl group?

Mei Wang replied: Because the diiron complex with a $\mathrm{SCH}_{2} \mathrm{NHCH}_{2} \mathrm{~S}$ bridge is very unstable, which thwarts the catalytic study of this complex. Although complex 1 has a nitrogen in the center of the S-to-S bridge, this nitrogen connecting to a phenyl group is a very weak base and cannot act as a proton relay during the catalytic process. In this case, both catalyst 1 and complex 2 cannot be protonated at the S-to-S bridge prior to reduction, and the catalytic performance of 1 and 2 can be compared to give information about the influence of the mercapto-functionalized $\beta$-CD on the HER.

Seigo Shima asked: In the native [FeFe]-hydrogenase, the $\mathrm{NH}$ at the bridge structure on the FeFe site is considered as a part of the catalytic site. I would like to know why such compounds that connect a large molecule at the nitrogen site can function as $\mathrm{H}_{2}$ forming catalysts. Does this compound catalyze $\mathrm{H}_{2}$ production using a mechanism which differs from that used in [FeFe]-hydrogenase?

Mei Wang responded: Yes, complex 1 catalyzes proton reduction to $\mathrm{H}_{2}$ in a different mechanism to that catalyzed by [FeFe]-hydrogenase. Firstly, complex 1 has a nitrogen in the center of the S-to-S bridge, which cannot play the role of a proton relay as the $\mathrm{NH}$ in [FeFe]-hydrogenase. Secondly, all diiron hydrogenase models reported to date undergo the $\mathrm{Fe}^{\mathrm{I}} \mathrm{Fe}^{0}$ oxidation state in the initial step of the catalytic cycle, while the $\mathrm{Fe}^{\mathrm{I}} \mathrm{Fe}^{0}$ oxidation state has not been found in the catalytic cycle of the [FeFe]-hydrogenases. 
Tomoaki Takayama asked: In the photocatalytic reaction using the diiron complex incorporated with $\beta$-cyclodextrin, it is essential to replace the ligand such as $\mathrm{CO}$ with the reactant such as an $\mathrm{H}_{2} \mathrm{O}$ molecule. Since the $\beta$-cyclodextrin covers the diiron complex catalyst, it is not easy to produce $\mathrm{H}_{2}$ by reduction of $\mathrm{H}_{2} \mathrm{O}$ molecules over the diiron complex. Do you have any information about the detailed mechanism of $\mathrm{H}_{2}$ production over the diiron complex incorporated with $\beta$-cyclodextrin? Did you determine a CO molecule disconnected from the diiron complex?

Mei Wang answered: For proton reduction to $\mathrm{H}_{2}$ or the reverse reaction catalyzed by natural [FeFe]-hydrogenases buried in protein environment, the ligand either $\mathrm{CO}$ or $\mathrm{CN}^{-}$at the $2 \mathrm{Fe} 2 \mathrm{~S}$ subcluster does not need to be replaced by $\mathrm{H}^{+}, \mathrm{H}_{2} \mathrm{O}$, or $\mathrm{H}_{2}$ molecules. The configuration of the $2 \mathrm{Fe} 2 \mathrm{~S}$ subcluster is very flexible. During the redox reaction, the $\mathrm{Fe}(\mathrm{CO})_{2}(\mathrm{CN})$ moiety could rotate to form a bridged $\mathrm{CO}$ and leave an open site for coordination of reactant, $\mathrm{H}^{+}, \mathrm{H}_{2} \mathrm{O}$, or $\mathrm{H}_{2}$ molecules. Although there are many reports on the electro- or photochemical hydrogen production catalyzed by bioinspired $2 \mathrm{Fe} 2 \mathrm{~S}$ models, the detailed mechanism for proton reduction to $\mathrm{H}_{2}$ or reverse reaction catalyzed by synthesized $2 \mathrm{Fe} 2 \mathrm{~S}$ complexes are currently not clear. In our $2 \mathrm{Fe} 2 \mathrm{~S} / \beta$-CD-6-S-CdSe assembly, one of the $\mathrm{Fe}(\mathrm{CO})_{3}$ moieties is outside of the $\mathrm{CD}$ cap, and could rotate to leave an open site for the coordination of the reactant as the natural $2 \mathrm{Fe} 2 \mathrm{~S}$ subcluster does.

Michael Wasielewski asked: When you compare the performance of catalysts with and without the p-aminophenylsulfonate group, there is very little difference. The presence of the electron-withdrawing sulfonate group on the aniline base dramatically reduces the basicity of the amine, thus making it ineffective as a pendant base to deliver the proton to the Fe in the complex. Please comment.

Mei Wang responded: Yes, the $\mathrm{N}$ in the aniline moiety with an electronwithdrawing group is a weak base, and it cannot be protonated in a $\mathrm{pH} 4.5$ aqueous solution. The present research was to explore the effect of mercaptofunctionalized $\mathrm{CD}$ on the performance of the 2Fe2S complex/CdSe QDs system. We chose $\left.\left[\left\{\beta-\mathrm{SCH}_{2} \mathrm{~N}\left(\mathrm{C}_{6} \mathrm{H}_{4}-4-\mathrm{SO}_{3} \mathrm{Na}\right) \mathrm{CH}_{2} \mathrm{~S}-\beta\right\} \mathrm{Fe}(\mathrm{CO})_{3}\right\}_{2}\right]$ as a catalyst to include into the cavity of $\mathrm{CD}$. To make a comparison, we used $\left[\left\{\beta-\mathrm{SCH}_{2} \mathrm{CH}_{2} \mathrm{CH}_{2} \mathrm{~S}-\beta\right\}\right.$ $\left.\left\{\mathrm{Fe}(\mathrm{CO})_{3}\right\}_{2}\right]$ as a reference catalyst which cannot include into the cavity of CD. Although both of these $2 \mathrm{Fe} 2 \mathrm{~S}$ complexes have no internal base that can act as a proton relay, their electrocatalytic and photocatalytic activity for proton reduction have been reported previously. In our studies, we found that both of these $2 \mathrm{Fe} 2 \mathrm{~S}$ complexes were catalytically active for $\mathrm{H}_{2}$ generation when combined with 6-S-CD-CdSe QDs in $\mathrm{pH} 4.5 \mathrm{H}_{2} \mathrm{~A}$ solution under illumination. It is worth of note that the estimated apparent electron transfer rate from the excited CdSe to $\mathbf{1}$ is about an order of magnitude faster than that from QDs to 2, and the TON of 1 is 22 -fold higher than that of 2 under the same conditions. Please also see the response to question 327 .

Sang Ook Kang remarked: The system looks simple at first glance but is not indeed. How sure are you that the flank can go into the CD inside? The sulphonate group is hydrophilic and it is difficult to visualise this getting into the hydrophobic interior of the CD. Instead of taking it for granted that your photocatayst 
can get into the interior of the $\mathrm{CD}$, please check the fundamental spectroscopic data closely and assess whether they correspond well to your proposed catalyst embedment.

Mei Wang replied: Professor Darensbourg and co-workers reported the inclusion of $\left.\left[\left\{\beta-\mathrm{SCH}_{2} \mathrm{~N}\left(\mathrm{C}_{6} \mathrm{H}_{4}-4-\mathrm{SO}_{3}\right) \mathrm{CH}_{2} \mathrm{~S}-\beta\right\} \mathrm{Fe}(\mathrm{CO})_{3}\right\}_{2}\right]$ into the hydrophobic cavity of $\beta$-CD and determined the structure of this assembly by spectroscopic studies and single crystal X-ray diffraction analysis. ${ }^{1}$ This result clearly demonstrated that the $\mathrm{NC}_{6} \mathrm{H}_{4}-4-\mathrm{SO}_{3}$ unit can readily be included into the hydrophobic cavity of $\beta$-CD. In the present study, we used the same method and the same $2 \mathrm{Fe} 2 \mathrm{~S}$ complex as in Darensbourg's report, ${ }^{1}$ and only changed $\beta$-CD to $\beta$-CD-6-SH. We characterized the assembly of complex 1 and $\beta$-CD-6-SH by HRMS, ${ }^{1} \mathrm{H}$ NMR, and IR spectra, which gave experimental proof for the inclusion of complex 1 into the cavity of $\beta$-CD-6-SH.

1 M. Y. Darensbourg et al., J. Am. Chem. Soc., 2010, 132, 8870.

Leif Hammarström commented: I want to comment on the question raised by Professor Wasielewski. We have published (in 2004) a similar complex (bromo instead of sulphonate on the phenyl), and it was impossible to protonate the nitrogen. We used the bromo group for linking to a Ru-sensitizer, and we could not protonate the nitrogen. Still, we observed quenching of the Ru emission, probably via Dexter energy transfer. With your CdSe NPs you may manage to get electron to the catalyst and protonate the complex in the reduced form (on the FeFe bond). So it still works but maybe via a different mechanism than when you can protonate the bridge prior to reduction.

Mei Wang responded: I agree with this comment. If the central $\mathrm{N}$ atom of the S-to-S bridge is directly bonded to a phenyl group, especially to a phenyl bearing an electron-withdrawing substituent, the basicity of this $\mathrm{N}$ atom is apparently decreased. In this case, this $\mathrm{N}$ atom cannot act as a proton relay in the proton reduction reaction. Accordingly, the catalyst $\left[\left\{\beta-\mathrm{SCH}_{2} \mathrm{~N}\left(\mathrm{C}_{6} \mathrm{H}_{4}-4\right.\right.\right.$ $\left.\left.\left.\mathrm{SO}_{3} \mathrm{Na}\right) \mathrm{CH}_{2} \mathrm{~S}-\beta\right\}\left\{\mathrm{Fe}(\mathrm{CO})_{3}\right\}_{2}\right]$ (1) displayed the same CVs at $\mathrm{pH} 7$ and $\mathrm{pH} 4.5$ in aqueous solutions, indicating that the $\mathrm{N}$ atom of the $\mathrm{S}$-to-S bridge cannot be protonated under test conditions. The purpose of the present study is to explore the effect of mercapto-functionalized $\mathrm{CD}$ on the performance of the $2 \mathrm{Fe} 2 \mathrm{~S}$ complex/CdSe QDs system. Although both the catalyst 1 and the reference $\left[\left\{\beta-\mathrm{SCH}_{2} \mathrm{CH}_{2} \mathrm{CH}_{2} \mathrm{~S}-\beta\right\}\left\{\mathrm{Fe}(\mathrm{CO})_{3}\right\}_{2}\right]$ (2) have no internal base that can act as a proton relay, we found that they were catalytically active for $\mathrm{H}_{2}$ generation when combined with 6-S-CD-CdSe QDs in $\mathrm{pH} 4.5 \mathrm{H}_{2} \mathrm{~A}$ solution under illumination. Because the $\mathrm{N}$ atom in complex 1 cannot be protonated under the test conditions, the two catalysts, 1 and 2, may function via a similar mechanism, in which the protonation takes place at the iron center of the reduced catalyst. The comparative studies revealed that inclusion of a diiron catalyst into the cavity of CD can improve the photostability of the catalyst, but does not have considerable influence on the rate of electron transfer, and connecting QDs to the host-guest assembly of CD and diiron catalyst has significant influence on the rate of electron transfer and as a consequence, on the photocatalytic $\mathrm{H}_{2}{ }^{-}$ evolving efficiency. 
Yosuke Kageshima remarked: In the time course of the long period photocatalytic reaction (Fig. $5 \mathrm{~b}$ in the paper), the amount of evolved hydrogen became saturated after $10-15 \mathrm{~h}$. What is the origin of deactivation of catalyst?

Mei Wang answered: The post-analysis suggested that both complex 1 and $\beta$ CD-6-S-CdSe QDs are partially deactivated under photocatalytic reaction conditions over a period of a long time. Although the stability of complex $\mathbf{1}$ is apparently improved in the presence of $\beta$-CD compared to the case in the absence of cyclodextrin, the decomposition of the all-CO diiron catalyst over long-term illumination is still a dominant problem for the deactivation of the $1 / \beta-C D-6-S-C d S e$ system. In addition, TEM images of CdSe QDs before and after long-term photolysis indicated a certain extent of aggregation of CdSe QDs.

Yosuke Kageshima further asked: Did you check the samples after a long term reaction? Was the deterioration of catalyst caused by damage to the semiconductor, or detachment of light absorber, and is it possible to check this experimentally?

Mei Wang responded: Yes, we have detected the CdSe QDs in solutions before and after long-term photocatalytic reaction by TEM, which indicated the aggregation of CdSe QDs to a certain extent after long-term photolysis, presumably due to the dissociation of the capping reagent, mercapto-functionalized $\beta$-CD from the surface of QDs. Such detachment also leads to disentangling of the close interaction between the light absorber and catalyst. It is difficult to experimentally detect the detachment of the protecting group as it does not give an observable change in UV-vis spectra, and the concentrations of QDs and diiron catalyst are very low $\left(1.0 \times 10^{-5}\right.$ to $\left.10^{-6} \mathrm{M}\right)$ in $0.28 \mathrm{M} \mathrm{H}_{2} \mathrm{~A}$ aqueous solutions.

Yosuke Kageshima said: You used CdSe QDs as the semiconductor material in the present study. Is it possible to use alternative materials, such as another semiconductor or a bulky one (i.e. not $\mathrm{QD})$ ?

Mei Wang replied: For homogeneous photocatalytic systems, QDs are good light absorbers in terms of their uniform distribution in solution, their tunable band edge and strong absorption intensity. For future work to fabricate photocathodes, other bulky semiconductors could be used to integrate with diiron hydrogenese models.

Akihiko Kudo commented: Which is the reaction path for the electron transfer from CdSe to the catalyst in Fig. 3, through bonds, CD, just jumping, or something else?

Mei Wang answered: Currently it is not clear whether the electron transfer from CdSe to the catalyst is through space, bonds, $\mathrm{CD}$, or something else. One of the possibilities is when CdSe is excited, the thiol groups of the capping agent for stabilizing the QDs may act as hole scavengers to suppress the recombination of excited electron. Some literature reports have proposed a similar role for other thiol-functionalized capping agents linked to CdSe QDs, and recently Professor 
Hammarström and co-workers have found some experimental evidence for such a function of the capping agent of CdSe QDs.

Vincent Artero asked: You propose that the detachment of cyclodextrin is one reason for deactivation. Why not choosing a cyclodextrin with more than one thiol function to ensure more stable grafting?

Mei Wang responded: This is a very constructive suggestion, and is the continuing work that we are trying to do now. To improve the stability of the linkage between cyclodextrin and CdSe QDs, we are trying to use $\beta$-cyclodextrin bearing seven mercapto substituents on the edge of its small ring (which is commercially available) as a protecting reagent of CdSe QDs. We will try to make photocathodes using this strategy to get rid of sacrificial electron donors. It can be anticipated that such a multi-point linkage between cyclodextrin and $\mathrm{CdX}(\mathrm{X}=\mathrm{S}$, Se) QDs would be more stable under photoelectrocatalytic testing conditions.

Anthony Harriman opened discussion on the paper by Peter Brueggeller: Can you comment on how the catalyst acquires multiple numbers of electrons. I think this is a fundamental aspect of many such systems but the successive charging of the catalyst is rarely examined.

Peter Brueggeller replied: This is an interesting problem especially regarding the Os-Pd dyad presented in our paper. This problem is related to the question, whether reductive or oxidative quenching of the chromophore is the dominant mechanism. Especially for our dyad, both mechanisms could occur. Therefore the chromophore could deliver two subsequent electrons reducing $\operatorname{Pd}($ II) to $\operatorname{Pd}(0)$ at the WRC side. The change of oxidation numbers would then be Os(I) to Os(III) at the chromophore side. I agree that this possibility should be examined!

Anthony Harriman remarked: Your molecular dyad, which has an interesting and logical structure, is set up for one-electron photochemistry. How does the second electron reach the catalyst? As it stands, it looks like some kind of shortcircuiting would take place if you excite the species where the catalyst has acquired an extra electron.

Peter Brueggeller answered: I think that the species where the catalyst has acquired an extra electron stems from the initial reductive quenching of the chromophore by the sacrificial donor. This is a consequence of the excess of sacrificial donor present in solution. It means that the species with an extra electron still consists of Os(II). Therefore a subsequent oxidative quenching is possible producing an extra second electron needed for the production of $\mathrm{H}_{2}$.

Anthony Harriman said: Concerning your rather nice molecular dyad, do you see evidence for short-circuiting? I mean, after the first cycle you have one electron sitting on the catalyst while the sensitizer has been restored via the sacrificial agent. The excited-state sensitizer might abstract the extra electron, giving rise to the kind of short-circuiting I'm talking about. Have you seen this effect? 
Peter Brueggeller answered: From the CV measurements we know that the Os(I) and Os(III) states are easily accessible. So after reductive quenching of the sensitizer by the sacrificial donor a short-circuiting effect could deliver the necessary two electrons by subsequent oxidative quenching. For a dyad, our catalyst shows a good activity. It seems therefore likely that this short-circuiting occurs, since Pd(I) would be very unstable, if only one electron sits on the WRC. Of course this idea has to be proved by transient absorption in the future.

Vincent Artero said: My question is related to the two-component $\mathrm{Cu} / \mathrm{Co}$ system. In collaboration with Mei Wang and Licheng Sun, we reported a similar system in $2012,{ }^{1}$ using a cyclometallated iridium photosensitizer. The results were similar when a phosphine ligand was used but there is a marked difference in the absence of any phosphine ligand. In our case, under illumination, we could observe the signal of the $\mathrm{Co}(\mathrm{I})$ species. Using a copper-based photosensitizer, you cannot observe the formation of $\mathrm{Co}(\mathrm{I})$ in the resting state under continuous irradiation. Can you comment on the redox properties of your Cu-based dye with respect to the Ir dye previously used? Furthermore, we now have another dimension by which to study this system: we can vary the potential of the photosensitizer in the excited/reduced state $(\mathrm{Cu} v s$. Ir). The positioning of this redox processes vs. the potentials of the $\mathrm{Co}(\mathrm{II}) / \mathrm{Co}(\mathrm{I})$ and $\mathrm{Co}(\mathrm{II})-\mathrm{H} / \mathrm{Co}(\mathrm{II})-\mathrm{H}$ couples should be key to explaining the discrepancy between this work and the previous one in terms of observing $\mathrm{Co}(\mathrm{I})$ or not in the absence of a phosphine ligand. Higher consumption rate of the $\mathrm{Co}(\mathrm{I})$ intermediate compared to its formation rate might also account for the fact that it does not accumulate in the solution.

1 P. Zhang et al., Inorg. Chem., 2012, 51, 2115-2120.

Peter Brueggeller responded: I completely agree that the possibility to observe the Co(I) intermediate in the absence of phosphine ligand could depend on the kind of photosensitizer used. It seems likely that the Ir-chromophore has enhanced reduction properties compared with the Cu-photosensitizer in the excited states. Typically Cu-chromophores show a bathochromic shift, when compared with their Ir counterparts.

Vincent Artero commented: I agree with the fact that phosphines stabilize the cobalt complexes in the +1 redox state but these reduced complexes can also be prepared or characterized without axial phosphine ligands (see reports by E. Reisner, J. C. Peters or ours ${ }^{1-3}$ ). If $\mathrm{Co}(\mathrm{I})$ is a catalytically relevant intermediate, it gets protonated and the key active species is likely the $\mathrm{Co}(\mathrm{II})-\mathrm{H}$ species formed by reduction of a $\mathrm{Co}(\mathrm{III})-\mathrm{H}$ species. Can you comment on the stabilization of such species by phosphine ligands?

1. X. Hu, B. S. Brunschwig and J. C. Peters, J. Am. Chem. Soc., 2007, 129, 8988-8998.

2. M. R. J. Scherer, N. M. Muresan, U. Steiner and E. Reisner, Chem. Commun., 2013, 49, 10453-10455.

3. N. Kaeffer, J. Massin, C. Lebrun, O. Renault, M. Chavarot-Kerlidou and V. Artero, J. Am. Chem. Soc., 2016, 138, 12308-12311. 
Peter Brueggeller replied: In my opinion there is a problem regarding the simultaneous presence of phosphine ligands and $\mathrm{Co}(\mathrm{III})-\mathrm{H}$ species, since it is wellknown that phosphines are oxidised by $\mathrm{Co}(\mathrm{III})$. On the other hand it is certainly possible that $\mathrm{Co}(\mathrm{II})-\mathrm{H}$ species are stabilized by phosphines. ${ }^{1}$ It seems likely that the oxidative addition of protons to $\mathrm{Co}(\mathrm{I})$ could produce a $\mathrm{Co}(\mathrm{III})-\mathrm{H}$ species in the absence of phosphines. However, the presence of phosphines stops the oxidation of $\mathrm{Co}(\mathrm{I})$ already in the $\mathrm{Co}(\mathrm{II})$ state.

1. M. Fessler et al., Dalton Trans., 2009, 8, 1383-1395.

Mei Wang asked: In your binuclear photocatalysts, the Ru and Pd moieties are connected by a 4-membered ring. Does the rigidity of this 4-membered ring play a key role in the catalytic activity and the stability of the photocatalyst? If we change this 4-membered ring into a more flexible and more stable 6-membered ring, will that influence the stability and the activity of the photocatalysts?

Peter Brueggeller responded: Due to the template assisted synthesis of the 4membered ring, it was not possible to replace 4-membered rings by 6-membered rings. However, it was possible to introduce an all trans configuration at the cyclobutane ring. This had a beneficial effect on the behaviour of the chromophore and the activity of the photocatalyst.

Joshua Karlsson opened a general discussion on the papers by Licheng Sun, Shigeyuki Masaoka, Mei Wang and Peter Brueggeller: How do we evaluate turnover number (TON) as a description of catalyst performance? Comparisons in the papers show massive increases, but these are not against a known standard. What numbers are we aiming for with a good TON? Is the catalyst with the highest TON necessarily always the best?

Mei Wang responded: The TON values reported by different groups were obtained under different conditions, such as different concentrations of catalyst, different $\mathrm{pH}$ values, different temperatures, different electron donors or acceptors, different light sources, and so on. Therefore, we cannot simply evaluate the HER and OER catalysts by directly comparing the TON values of catalysts. Only the TON values that were measured under identical conditions can be used to make a comparison of related catalysts. The catalyst with the highest TON is not always the best. In addition to TON, there are many other criteria for evaluating a catalyst, such as TOF, solar-to-hydrogen conversion efficiency, required reaction conditions, cost of catalyst, scalability, environmental influence, and so on.

Peter Brueggeller responded: Of course we have checked our photocatalytic device against known standards, giving the same published results. I think regarding the reductive side of water splitting a good TON is at the moment about 1000 or more for intermolecular systems. Additionally there is the problem of the costs of a given system.

Shigeyuki Masaoka responded: TON values can be one of benchmarks when we evaluate the performance of our catalyst. However, we need to consider other parameters such as overpotential, stability, cost, and scalability of the catalysts for 
practical use. I think we cannot determine "the best catalyst" only from TON value, and should evaluate the performance of the catalyst in a more comprehensive way.

Can Li asked Peter Brueggeller regarding his paper: What is the temperature effect on the activity? Have you observed similar hydrogen production activity? Have you ever investigated the temperature dependence on water oxidation?

Peter Brueggeller responded: We have checked the temperature effect on activity in the region ambient temperature up to about $323 \mathrm{~K}$. Regarding the dyad presented in the paper, this temperature effect seems to be beneficial for the observed hydrogen production activity. With regard to water oxidation we have not yet performed comparable investigations.

Can Li asked Mei Wang regarding her paper: Have you measured the Faradaic efficiency for photocatalysis on the QDs? What was the result?

Mei Wang responded: As it is a photocatalytic process, we have not measured the Faradaic efficiency, but we have measured the amount of $\mathrm{H}_{2}$ evolved from the solution during long-term photolysis and calculated the TON and TOF values for the systems, and we also measured the apparent quantum efficiency of the systems $(0.62 \%-3.19 \%)$.

Dogukan Hazar Apaydin asked: What are the reasons behind molecular catalysis being the preferred choice for academic research? Is it the ease of characterization? Do you see a future in a heterogeneous (catalysts immobilized on a surface) approach in electrocatalysis?

Mei Wang answered: The structures of molecular catalysts can be clearly characterized, and therefore this gives a chance to explore the mechanisms of HER and OER catalyzed by a molecular catalyst by means of various spectroscopy methods. This is one of the attractive advantages of molecular catalysts. In my opinion, the combination of molecular catalysts, especially those featuring selfhealing ability, with visible-light-absorbing semiconductor materials to fabricate photoelectrodes for OER and HER is one of the promising approaches to large-scale solar fuel production.

Peter Brueggeller replied: Certainly molecular precatalysts can definitely be characterized, mainly due to single crystal X-ray structure analysis. However, there is another point: molecular catalysts often show high TOF values, e.g. "Wilkinson's catalyst".

Nevertheless, I agree: excellent molecular catalysts should be immobilized on surfaces in order to enhance their stability. Furthermore, an electrocatalytic approach is very interesting, since this provides a possibility to overcome the problem of the sacrificial donors.

Alexander Kibler addressed Licheng Sun, Shigeyuki Masaoka, Mei Wang and Peter Brueggeller: What will be the direction of molecular catalysis in the future? When we appreciate the efficiency of natural photosynthesis, it is due to the 
precise molecular organisation and complete control of the first and second coordination spheres that is difficult to achieve in solution phase. Will the optimisation of catalytic metal complexes and their ligands hit a performance ceiling that can only be surpassed by hetero-organisation?

Mei Wang responded: Studies on molecular HER and OER catalysts are important because: i) molecular catalysts provide a way to explore the mechanisms of HER and OER; ii) bio-inspired molecular catalysts help us to have a better understanding of the enzymatic systems, PS II and PS I, and to learn from nature; iii) molecular catalysts can mimic the active edges of material catalysts, such as the side-on bound Mo(rv)-disulfide complex that mimics the surface of nanoparticulate $\mathrm{MoS}_{2}$; and iv) some molecular catalysts display not only very high activity but also self-healing ability, such as $\mathrm{CoP}_{i}$ and Brudvig's dinuclear Ir(III) Ir(III) catalyst, which are very promising catalysts for application in solar fuel production.

Peter Brueggeller answered: This is a typical problem of homogeneous versus heterogeneous catalysis. Some people believe that the optimisation of catalytic metal complexes and their ligands should be carried out as a first step. Then the same optimised catalysts could be immobilised on heterogeneous carriers like titaniumdioxide, silica or even MOFs. This could be the right way in order to mimic nature.

Shigeyuki Masaoka responded: As you suggested, the control over the second coordination spheres in addition to the catalytic centers is important. We have already started the project based on such a viewpoint and succeeded in improving the activity of the molecular catalyst for the water oxidation reaction by introducing the recognition sites to control the second coordination spheres into the ligand. ${ }^{1}$ Thus, I think that this kind of strategy to design ligands can be a new direction, which enables to utilize the second coordination spheres more effectively and improve catalytic activity tremendously.

1 M. Yoshida et al., Angew. Chem. Int. Ed., 2015, 54, 7981-7984.

Anthony Harriman commented: A problem that I see for the future development of the field is that too many systems are being pursued simultaneously by different research groups. There is not much critical comparison being attempted and each group looks to be following its own course. We have no general agreement on the types of catalysts, the reaction conditions or the preferred products. This is fine for academic research but is not so good for the development of a solar fuels industry. Perhaps it is time for some common, but fairly narrow, objectives to be set: for example, surely it is possible to rule out many metals as putative catalysts in the search for something workable. We are still working on ridiculously small scales, forty years after the initial experiments were initiated by Shilov, Graetzel, Lehn, Porter, etc. The continued use of sacrificial redox agents, in my opinion, is harmful to the field.

Vincent Artero addressed the delegates and speakers: To continue on the same track as Tony Harriman, I think we need to change directions and rather than 
discussing whether we need to select molecular $v s$. solid state systems or homogeneous vs. heterogeneous systems, I think we need to consider whether processes using sacrificial agents are relevant or not, and for what. In such systems a large amount of radicals are generated, which limits the stability of the systems. Also decomposition products of these sacrificial agents accumulate in solution. This is not the case when water is used as the source of electrons. In this case, the electrolyte/medium remains unchanged. To me, there is no point optimising a system under these conditions since the sacrificial donors or acceptors are also the source of decomposition. It will be relevant only when we will be able to use water as the electron and proton source in a tandem system. I therefore suggest to restrain the use of sacrificial agents for mechanistic studies, after checking that they do not interfere with the mechanism.

Mei Wang replied: I fully agree with this comment. Numerous photocatalytic systems, containing either sacrificial electron acceptors for the OER or donors for the HER, have been widely studied over past decade. On the basis of previous results, we should push the research in this field a step forward by putting more attention on the photosystems and photodevices which do not require sacrificial electron donors and acceptors for solar fuel production. Additionally, for some special purposes, e.g. for exploring the effect of a structural factor of the ligand or the influence of a secondary coordination sphere on the performances of HER or OER molecular catalysts, three-component photocatalytic systems containing sacrificial electron donors or acceptors are still one of the ways to make preliminary comparative evaluations before application of these catalysts and strategies to the fabrication of photoanodes and photocathodes.

Licheng Sun agreed: I totally agree with the comments and suggestions.

Anthony Harriman addressed Licheng Sun, Peter Brueggeller, Shigeyuki Masaoka and Mei Wang: We continue to employ sacrificial redox agents for isolating one of the half reactions. We have been doing this for a very long time and we all know there are major problems with this approach. The radicals that arise from the sacrificial agent go on to drive chemical reactions leading to water oxidation or reduction but these are rarely taken into account when considering the reaction mechanism. I think we fool ourselves into believing that these reagents are helpful when, in fact, they are really harmful. Do you think we have reached the stage where all use of sacrificial redox agents should be banned?

Mei Wang responded: I agree. On the basis of previous results and experiences, we should push the research in this field a step forward by putting more attention on the photosystems and photodevices which do not require sacrificial electron donors and acceptors for solar fuel production.

Peter Brueggeller replied: I do not think that all use of sacrificial redox agents should be banned. The oxidation of water is a four electron process. Otherwise also very reactive radicals would form like in the case of sacrificial donors. However, I agree that we should focus on the problem what a given sacrificial agent does. There are countries in the world, where water is more expensive than 
petrol. Therefore also an inexpensive sacrificial donor producing no harmful radicals could be a solution of this problem.

Shigeyuki Masaoka returned: I agree that the nature of sacrificial reagents can affect the catalytic reaction. We have noticed this point and have already studied the effect of $\mathrm{Ce}^{\mathrm{IV}}$ ions on the water oxidation reaction catalyzed by a ruthenium complex. ${ }^{1}$ What I learned from this study is that we need to carefully check the properties of sacrificial reagents and their effects on catalytic reactions. In my opinion, we do not have to ban all of these relevant techniques because the investigation of the catalytic activity using sacrificial reagents is a well-established and useful method.

1. M. Yoshida et al., Chem. Asian J., 2010, 5, 2369-2378.

Anthony Harriman addressed Licheng Sun, Peter Brueggeller, Mei Wang and Shigeyuki Masaoka: Following from my earlier comment, is anyone aware of any case where the radicals derived from the sacrificial redox agent (usually triethanolamine, triethylamine or persulfate) do not interfere in the subsequent electron-transfer chemistry? The situation might be even worse for carbon dioxide reduction where direct reaction between the amine and $\mathrm{CO}_{2}$ could form a carbamate. What do people think about the continued use of these materials?

Mei Wang answered: The water splitting with PEC cells is a promising approach for future application in a large scale. We should put more efforts onto the studies of various highly active and robust photoanodes and photocathodes, and onto the integration of spontaneous water splitting PEC cells.

Peter Brueggeller responded: The interference of radicals derived from the sacrificial redox agent in the subsequent electron-transfer chemistry could be responsible for the fact that usually only one kind of sacrificial donor works in a given system. So there is the need for inexpensive sacrificial donors, which do not give harmful radicals. Nature has chosen water for this purpose.

Shigeyuki Masaoka replied: As I mentioned in the answer to the previous question, the evaluation of catalytic activity by using sacrificial reagents is very useful and powerful technique if we know their properties well and use them carefully. However, as you indicated, the sacrificial reagents can play several roles in the catalytic reactions. Therefore, the detailed understanding of reaction mechanism, which may include the unexpected processes involving sacrificial reagents, is indispensable to evaluate the activity of the targeted catalyst correctly.

Hyunwoong Park addressed Licheng Sun, Shigeyuki Masaoka, Mei Wang and Peter Brueggeller: There is a need for a standardised global protocol for test devices. PV is a large factor and temperature is often a negative factor. When we make a standardised global protocol for testing, what temperature should we consider? Room temperature? Outside temperatures of $40 / 50{ }^{\circ} \mathrm{C}$ ?

Peter Brueggeller answered: I agree that in many cases temperature is a negative factor, where heating destroys the catalysts. However, for very stable catalysts 
a slight rise of temperature above ambient temperature $\left(40 / 50{ }^{\circ} \mathrm{C}\right)$ could be beneficial. Since solar radiation also produces heat, there is no need to cool the samples down. In any case, according to the talk of Prof. Licheng Sun it is important to know the temperature at which the reaction occurs. Regarding a standardised global protocol it is important to study temperature effects in detail.

Licheng Sun answered: We would like to suggest all authors to provide exact temperatures instead of using the words room temperature. For standardised protocol, maybe $25{ }^{\circ} \mathrm{C}$ can be considered.

Shigeyuki Masaoka answered: As you indicated, the establishment of a standardised global protocol for test devices is required and the operation temperature of the system is an important factor to be considered. However, the temperature to achieve best performance depends on the system because temperature can give both positive and negative effects. In my opinion, we do not have to set "standard temperature" to evaluate the performance of the devices. Rather, temperature should be treated as one of parameters to optimize the system.

Haruo Inoue said: I think that we are having a very good discussion about the temperature effects on enhancement as Professor Licheng Sun demonstrated. In addition to the positive enhancement, stability and durability of the systems also depend on the temperature during the actual operation. The most crucial thing would be that everyone should be encouraged to examine the temperature effects on his/her materials and systems, because reactions and processes with rather large activation energies could be surpressed at ambient temperature, while they would be exponentially enhanced at higher temperature and may cause serious problems.

Peter Brueggeller replied: I completely agree with this suggestion. We already have plans for a new photocatalytic device, where higher temperatures than ambient temperature can be adjusted.

Akihiko Kudo responded: I agree. Temperature effects are important. Temperature gave positive and negative effects when we examined it for Zschematic water splitting in suspension and the effects depended on the system. So, the temperature should not be unified for standardization as well as the amount of photocatalyst. Efficiency should be compared after optimizing the experimental condition for each system: How much of the $\mathrm{H}_{2}$ or CO reduction product is obtained under the optimized experimental condition using a solar simulator of a standard light source will bring a practical comparison of the efficiency as proposed in previous literature. ${ }^{1}$

1 A. Kudo and Y. Miseki, Chem. Soc. Rev., 2009, 38, 253.

Tohru Setoyama responded: Liquid phase reaction with $\mathrm{CO}_{2}$ will have some clear limits if the conversion rises. The supply of $\mathrm{CO}_{2}$ as a bubble will disturb the reaction because the access of water to the catalyst will compete with the diffusion of $\mathrm{CO}_{2}$ and solubility of $\mathrm{CO}_{2}$ in water which is limited. For these reasons, if 
pursuing the $\mathrm{CO}_{2}$ conversion by photocatalysis, the vapor phase reaction will be the final answer, so a higher temperature is preferable.

Young Soo Kang replied: Temperature is one of the critical parameters for $\mathrm{CO}_{2}$ reduction and water splitting reactions because it critically affects the kinetics of the reaction by achieving different activation energies of the redox reaction for the electron transfer and also adsorption and desorption of $\mathrm{CO}_{2}$ and other reactants from the surface of catalysts. It should be studied more systematically for the specific parameters such as electron transfer reaction and adsorption and desorption of reactant molecules.

Dogukan Hazar Apaydin addressed Anthony Harriman and Vincent Artero: I guess heterogeneous catalysis (where catalyst material is immobilized on the surface) might have an advantage in the case of liquid products. It may be the case that the characterization of molecular catalysts are easier compared to heterogeneous but when we have a liquid product one must also think of the separation process and energy requirements if the end goal is application on a larger scale.

Anthony Harriman responded: Yes, you are quite correct to stress the importance of the separation of the product from reactant. In the case of a liquid product, it will surely be necessary to recover the catalyst. This might also open a route for repair or regeneration of the catalyst. At the moment, we simply add more material when the reaction slows down. Perhaps we need to consult more with chemical engineers.

Alexander Kibler addressed Licheng Sun, Shigeyuki Masaoka, Mei Wang and Peter Brueggeller: When we evaluate the performance of catalysts there is an explicit academic focus on turnover number and turnover frequency. However for these to become economically viable alternative energy solutions they need to have appreciable stability over the timescales of months instead of hours. Should there be a shift in focus towards optimising stability and self repair mechanisms over catalytic activity to deliver catalysts that can be attractive towards industry?

Mei Wang replied: Activity, stability as well as low-cost are three crucial criteria for photocatalytic systems and devices from the application point of view. We cannot emphasize only one point and slight other points. Molecular catalysts that feature not only high activity for OER and HER but also self-healing property are very promising catalysts and are eagerly pursued by many researchers in recent years. $\mathrm{CoP}_{i}$ is a representative of such catalyst. The development of more catalysts that feature self-healing property is an attractive and challenging work.

Peter Brueggeller responded: This has certainly to do with the famous sentence of R. H. Grubbs: "Catalysis is always a battle between reactivity and stability". So I think we have to keep in mind all three factors:

1) Optimal TON or TOF.

2) Long stability.

3) Possible self repair of the catalysts. 
Shigeyuki Masaoka replied: I agree with your comments. The long-term stability of catalysts is indispensable for practical use. The self-repairing process of molecular catalysts is quite interesting and an important concept for this purpose, and could be the next breakthrough in this field.

Johannes Ehrmaier addressed Licheng Sun, Shigeyuki Masaoka, Mei Wang and Anthony Harriman: In my opinion, the price of produced fuel will be the decisive factor on whether an artificial photosynthesis system will be used for large scale applications. Should we not consider from the beginning that in the end the system has to be cheap and scalable?

Anthony Harriman responded: I'm less certain that price of the fuel has to be the main consideration from the beginning. I don't think we should be too preoccupied with costing until we have a large-scale system in operation. I'd be happy to see the photochemical production of speciality compounds-not necessarily fuels-as long as the scale is sufficiently impressive. I do have real concerns about the scalability and the longevity of the systems that are being proposed. It will not be easy to get industry serious about our work until we can compete with heterogeneous catalysis. What would help, is some kind of general consensus on what would be the best range of products: at the moment there is a bewildering variety of processes and materials being tested but these are rarely compared or contrasted with related systems. I think this is quite wasteful.

Mei Wang replied: Just as I have said before, activity and stability as well as low-cost and scalability are crucial criteria for photocatalytic systems and devices from the application point of view. We cannot emphasize only one point and slight other points. We have to balance these points. Recently, Brudvig and coworkers reported a binuclear $\operatorname{Ir}(\mathrm{III}) \operatorname{Ir}(\mathrm{III})$ catalyst, which is highly active and robust for OER. Modification of the surface of ITO or metal oxide semiconductors with a small amount of such catalyst, only one monolayer of molecules, gave highly active and stable anode or photoanodes for OER. This successful example shows that if only a small amount of catalyst is needed to fabricate highly active and stable electrodes or photoelectrodes, and the cost of solar fuel production devices containing noble metal catalysts can be controlled to an acceptable price.

Shigeyuki Masaoka responded: This is an important point. So far, an enormous number of molecular catalysts have been reported and we gradually become able to design catalysts on demand. Therefore, we need to start considering cost and scalability of the final system when we design new catalysts. It should be also noted that high-value added molecules can potentially be produced by molecularbased systems, and such a system should be constructed in addition to the conventional artificial photosynthesis system.

Richard Cogdell commented: Trying to calculate costs of possible solutions now is very hard. Remember how bulky and expensive the first transistor was? Now we have very cheap nano-transistors that would have been impossible to imagine when the first transistor was produced. I expect the same will be true for solar fuel solutions once the engineers get to work on them. 
Jian-Ren Shen replied: Yes, I completely agree.

Anthony Harriman commented: The most significant photochemical process, in my opinion, would be the reduction of nitrogen to ammonia. If we could do this under ambient conditions, it would be a major step forward and would establish the credentials of artificial photosynthesis as a serious contributor to renewable energy. I would go so far as to say that, if starting again, I would focus exclusively on this topic.

Akihiko Kudo responded: I agree to that $\mathrm{N}_{2}$ fixation is an attractive and challenging topic as well as water splitting to generate $\mathrm{H}_{2}$ and $\mathrm{CO}_{2}$ fixation. We should still continue to work on water splitting and $\mathrm{CO}_{2}$ fixation because these reactions are not completed yet.

Tohru Setoyama responded: From an industrial view point, the conventional HB process has established very well. Its productivity is excellent and catalyst life is almost eternal. There is a terribly long road toward establishing some technology which will work at ambient conditions. Therefore it may be more realistic to utilize solar hydrogen for $\mathrm{NH}_{3}$ synthesis in some improved HB type catalyst. In Japan, a new catalyst for $\mathrm{NH}_{3}$ synthesis is a hot topic. For example, a $\mathrm{Ru} / \mathrm{Ca}\left(\mathrm{NH}_{3}\right)_{2}$ catalyst shows a very high catalytic performance under $573 \mathrm{~K}$ at moderate pressure. I do not agree with the idea of utilization of $\mathrm{NH}_{3}$ as a fuel. It should be regarded in the same category as nuclear. If an accident happened, this would bring a disaster.

Flavia Cassiola remarked: It is exciting to have a strong support of processes that we could explore to establish the credentials of artificial photosynthesis as a serious contributor to renewable energy. We can't deny that reducing nitrogen to ammonia under ambient conditions will be a game changing technology. The energy demand for the growing global population cannot afford abandonment of any source that we can responsibly explore to provide energy to everyone. Reduction of nitrogen to ammonia is as important as reduction of carbon dioxide for solar fuels. Research should be encouraged and supported and we should plan for a session in artificial photosynthesis meetings to bring together the scientists (academia and industry) doing work on this process.

Vincent Artero opened discussion of the paper by Michael Wasielewski: You mention a $43 \mu$ s lifetime for the reduced species. I understand that this is sufficient for the chemical reaction to proceed but what about the expected delay between the absorption of two photons. In a dye-sensitized solar cell, a dye can capture a photon every second under solar irradiation.

Michael Wasielewski replied: These molecular triads are designed to make it possible to observe each step of the photo-driven catalytic mechanism, so that the photons are provided by laser pulses, which can provide photons at an adequate rate.

Etsuko Fujita queried: In your paper, you reported a molecular triad with the Py-DPA-NDI moiety directly coordinated to the rhenium centre of $\operatorname{Re}(\mathrm{dmb})(\mathrm{CO})_{3}$. 
Once the triad is reduced during photochemical $\mathrm{CO}_{2}$ reduction, the Py-DPA-NDI moiety dissociates from the rhenium centre. I am very pleased that you discussed a complex where the Py-DPA-NDI moiety is now attached to the dmb ligand. Your triad seems to have a quite complicated IR spectrum. In your transient IR experiments for $\mathrm{CO}_{2}$ reduction, can you see changes in the IR bands associated with intermediates such as the $\mathrm{Re}-\mathrm{CO}_{2}$ adduct in the 1500 to $1700 \mathrm{~cm}^{-1}$ region?

Michael Wasielewski answered: The carbonyl region of the transient IR spectrum is well-resolved and we have not found any difficulty in identifying and assigning specific carbonyl features.

Leif Hammarström commented: I like the approach you present. One problem for two-pulse excitation is that you need to have a good charge separation yield to get a reasonable probability of achieving double excitation. Were you able to quantify the quantum yield of the full charge separation in these systems?

Michael Wasielewski answered: The quantum yield for reduction of the bipyridine in the Re complex is $87 \%$.

Devens Gust asked: Would you envision using these types of systems for solar fuel production? If so, in general terms, how would this be done?

Michael Wasielewski responded: This system serves as a prototype for comparable systems that can be attached to a p-type semiconductor which will provide the electrons necessary to reduce NDI to its radical anion, thus providing a continuous source of radical anions that can be photo-excited to drive the carbon dioxide reduction catalyst.

Richard Cogdell asked: How well does your system work in the presence of oxygen?

Michael Wasielewski responded: Not well. The radical anions are oxidized back to the neutral species in the presence of oxygen.

Richard Cogdell queried: What concentration of carbon dioxide is needed?

Michael Wasielewski replied: Assuming diffusion controlled binding of carbon dioxide to the complex, when the photosensitizer is bound to the Re complex via the bipyridine, millimolar concentrations of carbon dioxide should be adequate.

Richard Cogdell commented: Then you are saying that practically it needs to be anaerobic?

Michael Wasielewski responded: Yes, these systems must be operated anaerobically.

Peter Summers remarked: In the complex linked through the bipyridine ligand (shown in the presentation) and the complex linked though axial coordination to the 
Re (in the paper) there is a difference in the charge associated with the Re moiety, does this help explain the difference in charge separation lifetime measurements?

Michael Wasielewski responded: No, the difference in charge separation lifetimes is largely due to the stronger electronic coupling between the photosensitizer and the complex when they are linked through covalent attachment at the bipyridine.

Etsuko Fujita commented: Your new triad is a very promising system for investigating photo-driven electron transfer and photochemical $\mathrm{CO}_{2}$ reductions. Have you tried electrochemical $\mathrm{CO}_{2}$ reduction with your triad? If so, did you observe CO formation? Do you have any idea how fast CO formation is?

Michael Wasielewski answered: I agree that testing photosensitizer-catalyst combinations for electrocatalytic activity to make sure that the activity of the catalyst has not been diminished by attachment of the photosensitizer is very important. We have not yet investigated the electrocatalytic properties of this system, but we intend to do so in the very near future.

Haruo Inoue asked: I am impressed with your beautiful analysis of the time constants etc. You showed two types of diad molecules, one contains one light harvesting part connected with Re complex and the other has a Re complex that is sandwiched by and connected with the two light harvesting chromophores. I understand the concept of the designing molecular systems, but experimentally is there any difference between them in observing transient species?

Michael Wasielewski replied: Thank you. The system with one radical anion photosensitizer attached to the Re complex is designed to perform a one-electron reduction of the Re complex, while the system with two radical anion photosensitizers attached to the Re complex is designed to transfer two electrons sequentially into the Re complex, thereby producing the state of the complex thought to bind carbon dioxide to begin the catalytic cycle. The latter experiment will be accomplished with two sequential laser pulses, one for each radical anion photosensitizer, which should result in one complete turnover of the Re complex in which we can observe all intermediates in real time. We have already demonstrated this type of a two sequential laser pulse experiment in purely organic multiple donor-acceptor systems, but as yet have not finished synthesizing the two photosensitizer Re complex.

Devens Gust commented: In catalytic applications, after the first photon has been absorbed by the dye anion and reduced the catalytic metal center with one electron, would absorption of a photon by any neutral naphthalene sensitizer lead to unwanted recombination reactions with the partially reduced metal?

Michael Wasielewski replied: No, because the neutral photosensitizer, NDI in this case, absorbs only in the UV region. 
Anthony Harriman queried: If the first excitation directs an electron to the bipyridine unit, this might affect the ability to send the second electron to the same unit. The presence of the electron on the bipyridine should affect the thermodynamics for the second electron transfer and might even change the mechanism. Do you have information on the successive excitation steps.

Michael Wasielewski answered: This is an interesting idea. We are currently carrying out those experiments, but have nothing as yet to report.

Etsuko Fujita opened discussion of the paper by Vincent Artero: The reductive quenching of the Ru excited state of your ruthenium-copper dyad seems to be quite slow even though you added large amounts of TEA. Why is it so slow?

Vincent Artero responded: Indeed the light-driven formation of the $\mathrm{Cu}(\mathrm{I})$ complex under irradiation and in the presence of a sacrificial electron donor is slow. This indicates a very low quantum yield of the process. Reductive quenching of $\mathrm{Ru}^{*}$ by TEA is reported in the literature to be inefficient, even at high concentrations of quencher.

Etsuko Fujita remarked: Did you try time-resolved experiments with your dyad?

Vincent Artero answered: We actually performed femtosecond and nanosecond time-resolved experiments and we have been unable to observe the signature of the $\mathrm{Cu}(\mathrm{I})$ species. As explained in the manuscript, we favor rapid electron transfer from the excited Ru moiety to the copper center, followed by an even faster recombination process. This pathway is fully unproductive but is highly competitive to reduction of the $\mathrm{Ru}^{\mathrm{III}}$ center by TEA. All together, these features explain the very low quantum yield observed for light-driven copper(II) reduction.

Devens Gust commented: You mentioned that the copper catalyst used for the click chemistry caused problems. Did you examine any other catalysts or procedures for the click reaction?

Vincent Artero answered: In our initial study dealing with the attachment of cobalt diimine-dioxime complexes onto carbon nanotubes, ${ }^{1}$ we use the copperfree AAC procedure developed by Bertozzi and relying on strained cyclooctyne. However, this introduces a quite flexible bridge, which is not very suited to study intramolecular processes. We also attempted the Ru-catalyzed azide-alkyne cycloaddition, without success (the experimental conditions being too harsh for our system).

1 E. S. Andreiadis, P. A. Jacques, P. D. Tran, A. Leyris, M. Chavarot-Kerlidou, B. Jousselme, M. Matheron, J. Pecaut, S. Palacin, M. Fontecave and V. Artero, Nat. Chem., 2013, 5, 48-53.

Peter Brueggeller asked: In your work you described an unwanted transmetallation of $\mathrm{Co}$ by $\mathrm{Cu}$. To get back to the initial system you reported a back transmetallation of $\mathrm{Cu}$ by $\mathrm{Co}$. From a preparative viewpoint this seems to be 
tricky. Could you please discuss this effect in more detail. Does the direction of the transmetallation depend on the oxidation number? Is this in line with the HSAB concept?

Vincent Artero replied: Under reducing CuAAC conditions, copper displaces the cobalt center in the diimine-dioxime complex and is thus not active to catalyze the cycloaddition any more. Obtaining a cobalt complex from a copper diimine dioxime complex is possible if you use a large excess of a $\mathrm{Co}$ (II) salt and you bubble air into the mixture to form a $\mathrm{Co}(\mathrm{III})$ complex. Indeed, $\mathrm{Co}(\mathrm{III})$ has a higher affinity than $\mathrm{Cu}(\mathrm{II})$ for the diimine dioxime ligand. This has been described in a recent paper. ${ }^{1}$

1 N. Kaeffer, J. Massin, C. Lebrun, O. Renault, M. Chavarot-Kerlidou and V. Artero, J. Am. Chem. Soc., 2016, 138, 12308-12311.

Anthony Harriman asked: The triazole linkage is very convenient from a synthetic point-of-view but I seem to recall some slight confusion as to whether it is a good conduit for electrons and/or holes. Our earlier work with Fabrice Odobel, using porphyrin-bound polyoxometallates, indicated poor conducting properties for electrons-we saw through-space (not through-bond) electron transfer. I seem to remember that this finding was not supported by other work, but the evidence was not strong. Do you have any comment on how good the electron conducting properties of the linkage are? I think it would be useful to be clear on this point for future synthetic strategies.

Vincent Artero responded: We need to have a look at work by S. Campagna, F. Scandola et al. ${ }^{1}$ Obviously, this will depend on the molecular orbitals and we need to model this system using theoretical methods.

1 S. Campagna, F. Scandola et al., Chem. Soc. Rev., 2014, 43, 4005-4018.

Anthony Harriman remarked: Following on from my previous comment, might it be possible to exploit this effect to improve selectivity in terms of rates of forward $v s$. backwards charge transfer in molecular dyads?

Vincent Artero replied: Our conclusion is that the recombination is faster than the forward electron transfer. Again, we need to have a look at the molecular orbitals of the bridge. In the literature, the mode of connection of the triazole ring to the photosensitizer and to the acceptor unit has been extensively studied for porphyrin-based dyads and, in some examples, was shown to influence the recombination dynamics. ${ }^{1}$ Studying a similar $\mathrm{RuCu}$ dyad but with the opposite connectivity (i.e. CuAAC coupling between an azidesubstituted PS and an alkyne substituted CuDODOH complex) could thus be quite interesting.

1 D. M. Guldi et al., J. Am. Chem. Soc., 2011, 133, 13036-13054

Devens Gust said: In a multi-electron fuel production reaction, the first photon absorbed by the dye will partially reduce the catalytic center. In normal sunlight, absorption of a second photon for the second reduction step could take on the 
order of a second. Would there be a problem with stability of the partially reduced catalyst for this period?

Vincent Artero answered: This is a key point for the design of such systems. Indeed there is a need to develop catalysts that are quite stable in the semireduced states.

Elizabeth Gibson asked: These questions relate to the rate of electron transfer and emission quenching. What is the extent of the electronic coupling between the dye and the Cu complex? I notice that the dyad it is not fully conjugated all the way through the bridge, especially between the triazole and the copper. Do you have feeling for the preferred angles between the phenyl and imidazole or triazole? Are there crystal structures of related systems or DFT calculations?

Vincent Artero responded: The bridge is almost fully conjugated but we have a few saturated bonds within the diimine dioxime ligand itself. This may reduce the electronic coupling and be the cause for the limited quenching of fluorescence. We do not have a crystal structure of the $\mathrm{Ru}-\mathrm{Cu}$ assembly. Likely, the triazole ring is perpendicular to the diimine-dioxime ligand. Obviously, it would be very interesting to model this system using quantum chemistry methods.

Elizabeth Gibson remarked: How much quenching do you observe with the separate $\mathrm{Cu}$ catalyst and the dye rather than them bound together? This would be useful to demonstrate that it's definitely electron transfer mediated by the bridge.

Vincent Artero responded: We measured fluorescence of solutions containing separate $\mathrm{Cu}$ (II) complex and $\mathrm{Ru}$ dye at $10 \mu \mathrm{M}$ concentration. We do not see any quenching of fluorescence. This observation favors intramolecular electron transfer as the oxidative quenching process in the supramolecular $\mathrm{Ru}-\mathrm{Cu}$ species.

Devens Gust asked: The sensitizer dye is quenched to the extent of about $50 \%$. Because this sensitizer excited state has a fairly long lifetime, does this mean that the electron transfer from the excited sensitizer to the catalytic center is quite slow?

Vincent Artero answered: You may be right. A slow rate may be due to the fact that the pathway from the Ru center to the $\mathrm{Cu}$ site is not fully conjugated, as noted by Elizabeth Gibson.

Charles Machan opened discussion of the paper by Hitoshi Ishida: I have a question concerning the final mechanism of the paper, specifically the interconversion of carboxylic acid and formate. This should generally require an intermediate hydride. I am aware of some precedence for converting a carboxylic acid to a hydride, but this generally requires basic conditions or high CO pressure. For instance, if you treat $\mathrm{Fe}(\mathrm{CO})_{5}$ with two equivalents of $\mathrm{KOH}$ under aprotic conditions you can generate $\mathrm{HFe}(\mathrm{CO})_{4}{ }^{-}$. Your experimental conditions would not favor this type of mechanism, instead it would be more likely to cleave the $\mathrm{C}-\mathrm{OH}$ bond of the carboxylic acid in a polar solvent with a high concentration of proton donors. 
You invoke the mechanism from our 2015 paper in J. Am. Chem. Soc., ${ }^{1}$ but our argument was based on an electrochemical driving force altering the relative hydricities of the $\mathrm{Ru}$ hydride and the formate ion, which is not the case here. Working with analogous ruthenium bipyridine compounds, we observed that the first reduction of these compounds was a proton-coupled event. The formation of a hydride can explain many parts of this mechanism, but it is not explicitly invoked in yours. Although there are obvious differences between electrochemical and photochemical systems, it is not immediately clear why the first reduction should differ mechanistically. Do you have any insight on this? Unimolecular $\mathrm{CO}_{2}$ fixation to produce formate should require an intermediate hydride species. Such reactivity from formic acid is observed thermally when $\mathrm{Ru}(\mathrm{Cl})_{3}$ and formic acid are combined and heated, the $\mathrm{Ru}(\mathrm{II})$ polymer $\left[\mathrm{Ru}(\mathrm{CO})_{2}(\mathrm{Cl})_{2}\right]_{n}$ is produced, with formic acid providing reducing equivalents and $\mathrm{CO}$ through a decarbonylation reaction. As we have observed, the formation of a ruthenium hydride can produce both CO and formic acid with high kinetic preference; very little hydrogen is observed. Can you explicitly rule this type of mechanism out experimentally?

1 C. W. Machan, M. D. Sampson, and C. P. Kubiak, J. Am. Chem. Soc., 2015, 137, 8564-8571.

Hitoshi Ishida answered: In our reaction, we imagine that the hydride species would generate in the conversion from the carboxylic acid complex to the formate complex. We have previously reported the reaction mechanism in which the catalyst dimer would preferably yield formic acid. ${ }^{1}$ In this case, we consider that the neighboring metal center can accept a hydride to support the conversion from the carboxylic acid to the formate. Unfortunately, we could not detect the dimer catalyst because the strong absorption of $\left[\mathrm{Ru}(\mathrm{bpy})_{3}\right]^{2+}$ used as the photosensitizer overlaps the absorption in the visible absorption spectrum. In this work, we have carried out the reactions in ethanol/DMA instead of water/DMA, and we have not observed the catalyst concentration dependence in $\mathrm{CO} /$ formate selectivity. This suggests that no catalyst dimer is generated in ethanol/DMA. This may be a reason why the formate selectivity is quite low in these reactions.

I feel that the researchers in the USA prefer and believe in the hydride mechanism and many Japanese researchers conversely believe the carboxylic acid mechanism to be correct. I am not sure which European researchers prefer. The reason why we believe the carboxylic acid mechanism to be most likely is that the $\mathrm{CO}_{2}$ adduct generated by the reaction of a low-valent metal center with $\mathrm{CO}_{2}$ is reasonable. Additionally, the mechanism readily explains the $\mathrm{CO}$ formation under the more protic conditions. It also reasonably explains why the $\mathrm{CO}_{2}$ reduction does not accompany $\mathrm{H}_{2}$ production. Of course, I know that there are many works on an insertion of $\mathrm{CO}_{2}$ into a metal-hydride bond, while there are few works on the interconversion of carboxylic acid complex to formate complex.

I consider the possibility that there are actually many possible pathways for $\mathrm{CO}_{2}$ reduction. Depending on the reaction conditions, a different path may be taken. Our work is on photochemical reactions not electrochemical reductions. In the photo-induced reaction, the electron injection is very slow, while in the electrochemical ones the electrons rapidly inject into the catalyst. Considering the results that the reduction product selectivity strongly depends on a subtle difference in the reaction conditions, all the proposed reaction mechanisms may be correct. 
1 H. Ishida et al., Chem. Sci., 2015, 6, 3063-3074.

Leif Hammarström remarked: In your reaction scheme (Fig. 6) you have introduced a catalytic effect of protons on the pathway towards formate, from the $\mathrm{Ru}^{+}-\mathrm{COOH}$ species. However, to explain why the ratio of formate $v s$. CO increases with more ethanol, it is sufficient to see that the initial equilibrium between $\mathrm{Ru}^{+}-$ $\mathrm{CO}$ and $\mathrm{Ru}^{+}-\mathrm{COOH}$ is shifted towards the latter when water concentration is increased; addition of ethanol means addition of water. Therefore there is not necessarily a catalytic proton involved in pathway. Have I understood this correctly?

Hitoshi Ishida responded: We consider that ethanol, which has a very high $\mathrm{p} K_{\mathrm{a}}$, is too weak as a proton source to shift the first equilibrium from $\mathrm{Ru}^{2+}-\mathrm{COOH}$ to $\mathrm{Ru}^{2+}-\mathrm{CO}$, and therefore we draw the reaction with a dashed line in the scheme in Fig. 6. No one knows the details of the equilibrium reaction between $\mathrm{Ru}^{+}-$ $\mathrm{COOH}$ and $\mathrm{Ru}^{+}-\mathrm{CO}$ which are the one-electron reduced complexes. As you indicted, increasing the EtOH content possibly enhances the CO selectivity, but this idea would not be able to explain the differences in temperature dependence of the product selectivity in the different EtOH/DMA solutions. We envisage that the electron transfer rates also affect the $\mathrm{CO} /$ formate selectivity. Please be aware that increasing the EtOH content makes the electron transfer rates slow.

Leif Hammarström said: Your activation energy analysis gives detailed numbers of Eyring parameters etc. It is important to realise that this type of photoaccumulation experiment is different from the typical kinetic experiment where all reactants are added at $t=0$. Here, the rate of product formation you observe is given by the rate of photoexcitation multiplied by several yield factors for the successive reaction steps, i.e. by rate ratios instead of direct reaction rates. Your overall yields are typically below 10\%, which means that most of your absorbed photon and generated electrons are going elsewhere, in competing parallel reactions. Thus, in principle, an apparent activation energy for e.g. formate formation can be the result of a temperature-dependent competing reaction, even if formate production steps would be temperature independent. Therefore one has to interpret these apparent activation energies with caution: they are not activation energies based on reaction rates, but on quantum yields.

Hitoshi Ishida answered: We realize that the activation energy parameters we obtained are apparent. Particularly, it seems difficult to evaluate the entropy parameters, and therefore we have obtained the differences in activation parameters between $\mathrm{CO}$ and formate production, which should correspond the energy difference in the product selectivity.

As you indicated, the quantum yields of the catalyses are below $10 \%$. As the excited states of the photosensitizer are almost quantitatively quenched, we suppose that the major pathway to go elsewhere is the non-radiative deactivation, which is common in EtOH/DMA solutions. Another pathway may be a backelectron transfer from the reduced photosensitizer or the reduced catalyst to the oxidized electron donor. Although there are many pathways in the catalyses, the rate-determining steps are few. We suppose that the rate-determining step 
would reflect the activation parameters. Of course, we have to keep in mind that the values are not real but apparent.

Ferdi Karadas opened a general discussion of the papers by Michael Wasielewski, Vincent Artero and Hitoshi Ishida: How important is the distance between the chromophore metal site and the catalytic metal site? How is the electron transferred between these two sites? Is it through the ligand, or solution, or is some other mechanism involved?

Devens Gust responded: When an electron is transferred between two sites within a single molecule, the transfer almost always occurs through the bonds that link the two sites. This is because electron transfer requires some overlap between the wave functions of the initial and final states. Except in some unusual geometries, the overlap is generally largest when it occurs via the linker bonds.

Michael Wasielewski replied: The distance between the chromophore and metal complex is critical as is the nature of the chemical bonds between them because the electron transfer goes through the bonds.

Vincent Artero answered: This is a good question that has not been addressed in full yet and depends on the system. There is a general law for through-space electron transfer and the rate depends on the distance between both centers. In general the process is faster if electron transfer can proceed through bonds. You can find a lot of information in work by S. Campagna, F. Scandola et al. in Chem. Soc. Rev. ${ }^{1}$

1 S. Campagna, F. Scandola et al., Chem. Soc. Rev., 2014, 43, 4005-4018.

Hitoshi Ishida replied: The electron transfer happens when a reduced photosensitizer has a collision with a catalyst. The distance may be important but in the mixed systems we cannot control this. We suppose that the electron transfer would occur through space in the collision complex.

Ferdi Karadas addressed Vincent Artero, Michael Wasielewski, Devens Gust and Hitoshi Ishida: Are there any general criteria that should be considered for the design of the bridging ligand that connects the chromophore to the catalytic site in molecular dyads? For example, does it have to be conjugated?

Devens Gust responded: The orbital overlap required for electron transfer is generally greater through conjugated double bonds than through saturated linkages, but in some cases even saturated linkages are very effective. We must remember that a linkage that is very effective for forward electron transfer is also usually very effective for charge recombination (back electron transfer). Thus, for many applications, the linkage with the most overlap is not the best one to use.

Michael Wasielewski responded: Generally, electron transfer through conjugated spacer (bridge) molecules is faster than through non-conjugated spacers. The effects of the spacer structure on electron transfer rates is fairly well established. 
Vincent Artero replied: Here again, you can find a lot of information in work by S. Campagna, F. Scandola et al. in Chem. Soc. Rev. ${ }^{1}$ Generally, this depends on the molecular orbitals of the bridge. Just keep in mind that if a conjugated bridge increases the forward electron transfer rate, it also makes the recombination process easier and faster.

1 S. Campagna, F. Scandola et al., Chem. Soc. Rev., 2014, 43, 4005-4018.

Hitoshi Ishida responded: Connecting the photosensitizing site with the catalytic site by covalent bond would be effective because the collision between two sites is entropically advantageous. Professor Ishitani has synthesized many supramolecular photocatalysts and reported some good results. We are also working on such methods, and my student will give a poster presentation in the coming 2017 International Conference on Artificial Photosynthesis.

Devens Gust asked: I have often heard it stated that molecular catalysts such as the kinds we have all been studying can never be practical because they lack sufficient stability. What do you all think about that opinion?

Michael Wasielewski replied: Both ourselves and others have shown that atomic layer deposition provides an effective means of stabilizing molecular catalysts on semiconductor surfaces without destroying their catalytic activity.

Vincent Artero replied: Once you identify the reasons for instability, you can develop strategies to overcome these. There are two counter examples: organic solar cells and dye-sensitized solar cells contain molecular chromophores and they are quite stable under prolonged testing.

Hitoshi Ishida responded: I don’t agree that molecular catalysts always lack stability, but I partly agree that they are difficult for practical uses in many cases. However, they are often useful in providing information of reaction mechanisms which lead to the design of new catalysts.

Devens Gust addressed Vincent Artero, Mei Wang and Hitoshi Ishida: When people ask me about the viability of molecular systems, I often reply that the only currently practical system for solar fuels that we have is the one that supplies about $85 \%$ of our energy-photosynthesis-which is a molecular system.

Mei Wang responded: In my opinion, one of the promising applications of molecular catalysts is to integrate the highly active molecular catalysts for OER or HER with visible-light-absorbing semiconductors to fabricate hybrid photoanodes and photocathodes. The demonstrative example is the hybrid photoanode of dinuclear $\operatorname{Ir}(\mathrm{III}) \operatorname{Ir}(\mathrm{III})$ catalyst $/ \mathrm{Fe}_{2} \mathrm{O}_{3}$, which displays much higher activity than $\mathrm{IrO}_{x} / \mathrm{Fe}_{2} \mathrm{O}_{3}$ photoanode. Compared to solid material catalysts, molecular catalysts can be covalently or coordinately linked to the surface of semiconductor materials to form a light penetrable and electrolyte permeable molecular layer. 
Devens Gust commented: About 25 years ago I saw a demonstration of an organic LED display which had beautiful colors. However most people at that time said that OLEDs could never be practical because they were too unstable. Today we can go out and buy large screen OLED televisions. For another example, the photochromic molecules used in self-darkening sun glasses are very unstable under irradiation in solution, but they are quite stable in the commercial glasses due to the incorporation of various photoprotection systems. Finally, dye sensitized solar cells are practical devices with lifetimes suitable for commercial applications. These three examples show that molecule based systems and devices can indeed be practical.

Michael Wasielewski agreed: You are quite correct. I expect that encapsulation and photoprotection strategies similar to those that have made OLEDs a commercial reality will also be applied successfully to organic photovoltaics and other molecular systems for artificial photosynthesis.

Richard Cogdell asked: It was clear also this morning that specificity of the product from carbon dioxide reduction is a problem. How do you think this can be improved?

Michael Wasielewski responded: These Re tricarbonyl complexes generally favor CO formation over hydrogen.

Richard Cogdell asked: So which product would you choose?

Michael Wasielewski responded: The simplest product is CO or a mixture of $\mathrm{CO}$ and hydrogen (syngas) that can be employed directly in well-known catalytic processes for making chemical feedstocks and fuels.

Richard Cogdell commented: We will hear from Professor Nocera tomorrow about how he has used a bacterium to get the specificity of catalysis required. You just have to find the right organism and harness its catalytic capacity.

Flavia Cassiola queried: How do you imagine the private energy sector will contribute towards a viable artificial photosynthesis prototype? Do you think there are any contributions that could be explored within the energy sector expertise that we are not yet considering at the early stages of a PEC system?

Federico Franco opened discussion of the paper by Charles Machan: In the alternative catalytic mechanism for $\mathrm{CO}_{2}$ reduction to $\mathrm{CO}$ you proposed, you referred to a dimeric intermediate (denoted as F), containing a bridging $\mathrm{CO}_{2}$ molecule. Do you have any experimental evidence of this?

Charles Machan responded: The catalytic current shows a second-order dependence on catalyst concentration, which is indirect evidence of this. Etsuko Fujita and co-workers have characterized this species experimentally and subsequently published a computational examination of this mechanism which invokes similar intermediates. ${ }^{1}$ 
1 E. Fujita et al., J. Am. Chem. Soc., 2012, 134, 5180-5186.

Federico Franco said: In the paper the dimer disruptive effect of DMF is reported, introducing competitive H-bonds. Did you perform spectroelectrochemistry experiments in DMF, in order to prove that different transient species are actually formed in different solvents?

Charles Machan replied: The best evidence for the disruption of the hydrogenbonding effect comes from CV data, specifically that the peak catalytic current, and indeed the number and nature of reductive features, is wholly dependent on the strongly hydrogen-bonding nature of the amide groups. We did not obtain IRspectroelectrochemical data in $N, N$-DMF for the original paper. ${ }^{1}$

1 C. Machan et al., J. Am. Chem. Soc., 2014, 136, 14598-14607.

Leif Hammarström commented: I like the approach of the peptide-decorated carbon-reduction catalysts to shuttle protons more efficiently to the catalytic site. First, you see a clear increase in current with tyrosine vs. alanine. Have you been able to extract rate constants?

Charles Machan replied: Because of the complexity of the catalytic waveforms, the best we have been able to do is a qualitative comparison based on the difference between catalytic current and Faradaic current. This gives apparent turnover frequencies of $0.92 \mathrm{~s}^{-1}$ and $46 \mathrm{~s}^{-1}$ for the phenylalanine and tyrosine derivatives, respectively, in the absence of an external proton source. ${ }^{1}$

1 C. Machan et al., J. Am. Chem. Soc., 2016, 138, 8184-8193.

Leif Hammarström commented: I did not see that you deliberately added a proton source. Is trace water the source, or do you only see reaction of the protons that are already on tyrosine to start with? Have you done experiments
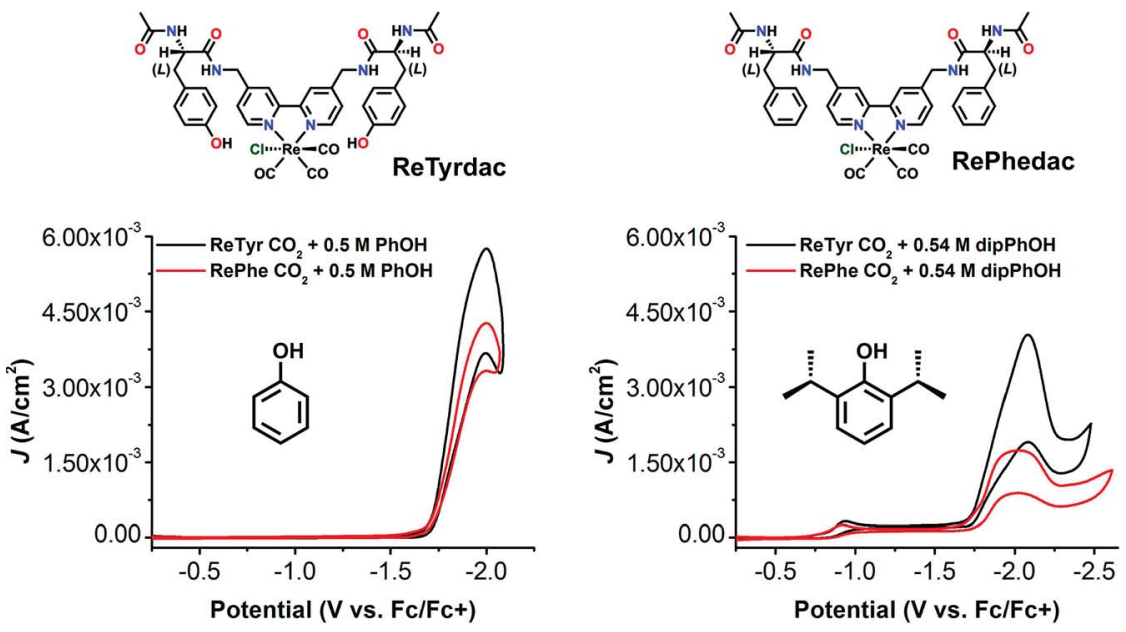

Fig. 1 Comparison of an excess of phenol with an excess of a kinetically limited proton source, diisopropylphenol. 
adding an excess proton source to exclude that differences in water content between samples affects the comparisons, and to shown that tyrosine really shuttles protons from a source, so that it is protonated again and does not just work once?

Charles Machan replied: The data in Fig. 1 show a comparison of an excess of phenol with an excess of a kinetically limited proton source, diisopropylphenol. These data show a greater difference in catalytic current is achieved with the kinetically limited proton source, which supports the existence of a proton relay effect.

Jose Martinez said: Could you comment on the advantages/disadvantages on incorporating these rhenium complexes into a peptide $v s$. a MOF or something else instead?

Charles Machan responded: The use of the peptide is advantageous because it accesses a bimolecular mechanism which is $250 \mathrm{mV}$ lower in overpotential as opposed to the unimolecular mechanism. The advantage of heterogenizing catalysts within metal-organic frameworks is that diffusion no longer becomes a factor. As we begin adding more amino acids to these catalysts, the rate of diffusion slows considerably. This is the limiting factor when it comes to this peptide-based catalyst design.

Can Li asked: I have an interest in bio-inspired systems, are there any bioprocesses which reduce $\mathrm{CO}_{2}$ to $\mathrm{CO}$ as in chemistry? Are there any natural photosynthesis processes that produce $\mathrm{CO}$ from $\mathrm{CO}_{2}$ reduction?

Charles Machan replied: There are several types of biological systems, such as RuBisCO or Acetyl-coenzyme A, which produce carbon monoxide from $\mathrm{CO}_{2}$. This is not utilized as an end product, however, but rather as an intermediate to further transformations.

Hitoshi Ishida asked: The pendent tyrosine interacts with the catalytic site by hydrogen bonding. The CV shows an enhanced cathodic current on the first reduction. I guess that the first reduction species of rhenium complex would not
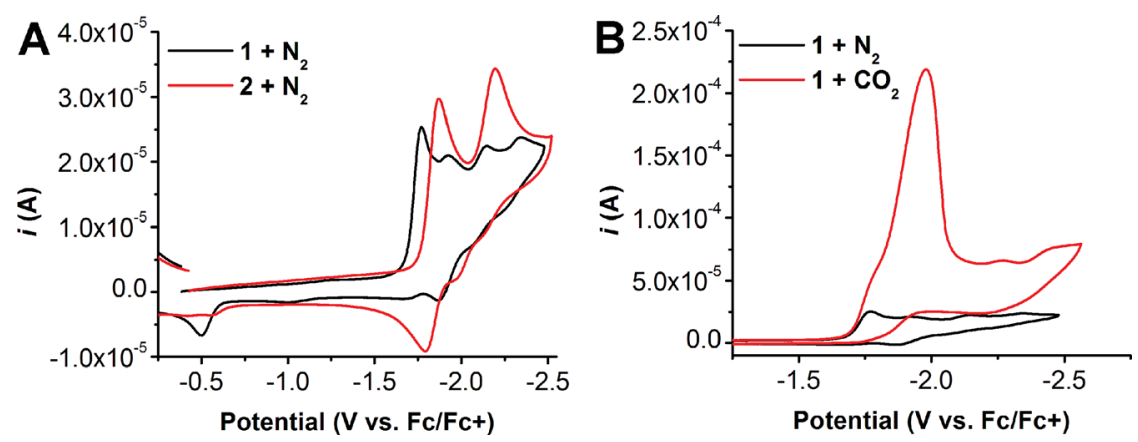

Fig. 2 Cyclic voltammetry under inert conditions where 1 is the amide-modified catalyst and 2 is the alkyl-modified catalyst. 
react with $\mathrm{CO}_{2}$. How do you explain the enhanced cathodic current at the first reduction potential?

Charles Machan responded: The cyclic voltammetry under inert conditions in acetonitrile actually shows four reduction features, which correspond to the stepwise reduction of two equivalents of the Re(bpy) catalyst until each have been reduced by two electrons. A catalytic current is observed at the second of these two reduction features because each of the two Re centers has been provided with one electron and the pair is now capable of capturing and then catalyzing the reduction of carbon dioxide.

This is shown in Fig. 2 where $\mathbf{1}$ is the amide-modified catalyst and $\mathbf{2}$ is the alkylmodified catalyst. The mechanism is explained in greater detail in the original paper. $^{1}$

1 C. Machan et al., J. Am. Chem. Soc., 2014, 136, 14598-14607.

Hitoshi Ishida commented: In your catalysis, the rhenium complexes dimerize with hydrogen bonding between the amino acid residues. When $\mathrm{CO}_{2}$ reacts with the catalyst dimer, isn't the hydrogen bonding disadvantageous?

Charles Machan replied: The catalyst dimer system achieves lower current density (and consequently turnover frequency) than the monomeric system, but can catalyze the reduction of carbon dioxide with $c a .250 \mathrm{mV}$ less overpotential. However, in the example with the tyrosine-modified catalyst system, turnover frequencies on the same order of magnitude as alkyl-modified catalysts can be obtained with no loss of product selectivity. ${ }^{1}$

1 C. Machan et al., J. Am. Chem. Soc., 2016, 138, 8184-8193.

Devens Gust said: This is a very nice example of the use of an amino acid to provide hydrogen bonding to the catalytic center. How far has the field as a whole come towards being able to design actual protein analogs to enzymes that provide the various "outer sphere" functions performed by natural enzymes?

Charles Machan responded: The most success with regards to design of artificial proteins generally relies on a combination of experimental and theoretical techniques. Through such methods, the field is capable of generating sequences which will bind metal ions and, although computationally expensive, predictive methods for folding are constantly improving. The limitation is that these methods are a macroscale approach to the question, as it is fundamentally difficult to predict if an artificial protein will exhibit catalytic behavior. There is still room for improvement in connecting molecular- and macroscale understanding through the secondary coordination sphere, or more specifically the 20 Angstroms surrounding a metal-based active site in an artificial system.

Sang Ook Kang opened discussion of the paper by Etsuko Fujita: Your photocataysts bear dual functionalities: sensitizer and catalyst. How do you control the activity? For example, by tipping one function over the other? 
Etsuko Fujita responded: The Ir complexes I presented are a few metal complexes that exhibit dual functionalities for $\mathrm{CO}_{2}$ reduction. Other well-known complexes are the family of $\operatorname{Re}(\mathrm{bpy})(\mathrm{CO})_{3} \mathrm{Cl}$ where bpy is 2,2'-bipyridine. First of all, these types of photocatalyst must have a long-lived excited state that is capable of separating charges (or of being reductively quenched by a sacrificial electron donor) in order produce the highly reduced species. The reduced species of these photocatalysts normally lose the coordinating $\mathrm{Cl}$ anion or solvent molecule to produce a vacant coordination site for an interaction with $\mathrm{CO}_{2}$. Furthermore, lowenergy pathways for $\mathrm{CO}_{2}$ reduction to $\mathrm{CO}$ and formate are 2-electron processes. Therefore, the one-electron-reduced species (or the $\mathrm{CO}_{2}$ adduct) must be stable enough to receive another electron.

Sang Ook Kang remarked: There are small alterations to the overall the Ir charge. I am wondering if on switching from the Ru to Ir center, how would you expect hydricity change?

Etsuko Fujita replied: It is difficult to answer your question. Hydricity depends on the reduction potential and $\mathrm{p} K_{\mathrm{a}}$ of the metal hydride. Without knowing these data, we cannot estimate the hydricity.

Devens Gust noted: My colleague Thomas Mallouk likes to say that iridium is not the least abundant element on earth, but only the least abundant stable element. What have you learned from studying these iridium systems that can help us design better catalysts that use more abundant elements?

Etsuko Fujita responded: I agree with Tom Mallouk. Our iridium complexes are quite durable, therefore, we can investigate the mechanism of $\mathrm{CO}_{2}$ hydrogenation. In order to increase catalytic activity, we placed $\mathrm{OH}$ groups near the iridium centre inspired by the iron guanylylpyridinol prosthetic group of [Fe]hydrogenase that catalyzes reversible hydrogen heterolysis. Since this biorelated design principle worked very well for iridium complexes, we applied it for $\mathrm{Cp}^{*} \mathrm{Co}$ complexes. However, the cobalt complexes easily lose the $\mathrm{Cp} *$ ligand in water and the catalytic activity was disappointingly low.

Vincent Artero asked: Can you clarify the sentence from the abstract “... $\left[\mathrm{Ir}(\mathrm{L} 3)(\mathrm{ppy})\left(\mathrm{NCCH}_{3}\right)\right]^{+}$cannot be reduced photochemically because the excited state reduction potential is only $0.52 \mathrm{~V}$." Do you mean that the exited state cannot react with the sacrificial electron donor? What is the oxidation potential of $\mathrm{Et}_{3} \mathrm{~N}$ ?

Etsuko Fujita responded: Yes, the excited state of $\left[\operatorname{Ir}(\mathrm{L} 3)(\mathrm{ppy})\left(\mathrm{NCCH}_{3}\right)\right]^{+}$does not react with $\mathrm{Et}_{3} \mathrm{~N}$. The oxidation potential of $\mathrm{Et}_{3} \mathrm{~N}$ is estimated to be $\sim 0.96 \mathrm{~V}$.

Dogukan Hazar Apaydin opened a general discussion of the papers by Charles Machan and Etsuko Fujita: At the end of their original paper, Lehn and coworkers suggested the use of other materials for bipyridine type of complexes. ${ }^{1}$ Strictly speaking, for bipyridine metal complexes is there a reason why we do not see many examples other than rhenium and manganese metal centres?

1. J. Hawecker, J.-M. Lehn and R. Ziessel, J. Chem. Soc., Chem. Commun., 1984, 328 
Etsuko Fujita replied: $\mathrm{Co}(\mathrm{bpy})_{3}{ }^{2+}, \mathrm{Ni}(\mathrm{bpy})_{3}{ }^{2+}$ and $\mathrm{Rh}(\mathrm{bpy})_{3}{ }^{3+}$ have been used as pre-catalysts for $\mathrm{CO}_{2}$ reduction. In order to react with $\mathrm{CO}_{2}$, the reduced species of these complexes must dissociate a coordinated bpy ligand. However, the catalytic activity of $\mathrm{CO}_{2}$ reduction is quite low due to strong binding of the produced $\mathrm{CO}$ to the reduced species leading to poisoning. Meanwhile, square-planar cobalt and nickel macrocyclic complexes have been successfully used for $\mathrm{CO}_{2}$ reduction.

Charles Machan answered: Bipyridine-based electrocatalysts are observed for other transition metals with carbonyl ligands: molybdenum, tungsten, ruthenium, and osmium. Other complexes with Rh and Ir contain multiple bipyridyl ligands and terpyridine and quaterpyridine have been found to be suitable ligand platforms for first-row transition metals. The difficulty is finding a heteroleptic ligand system which will favor CO loss at negative potentials and is stable under electrolysis conditions.

Michael Wasielewski asked: In designing a small peptide environment for the catalyst, can you build into the design a way to include important hydrogenbonding interactions without preparing what would essentially be a small protein?

Charles Machan answered: This addresses the heart of the basic question we are trying to answer. With smaller peptide sequences reactions can be more dynamic, but with increasing size with changes in diffusion it becomes harder to characterize these effects. At this point, we can provide a means for aggregation and proton sources for the active site. Incremental improvement requires that thorough experimental characterization be done with theoretical modeling in order to select the next step forward. We hope to build the kind of understanding you are describing through these studies.

Jose Martinez queried: At what point do we say, we learned enough from these non-earth abundant metals and begin to look at those that are more abundant? Or do we wait until astro-mining?

Charles Machan responded: The importance of this system is that we are studying how secondary effects can be used to control catalytic mechanism. In order to be able to do this, we need to be able to begin from a stable catalyst with a well-defined mechanism. This is less about the Re center and more about what type of control we can exert outside more traditional modification of sterics or electronics.

Etsuko Fujita replied: There is nothing wrong with using non-earth abundant metals for mechanistic investigations and learning new design principles for catalysts. Hopefully, we will find the materials we need on our planet. Or these non-earth abundant metal catalysts are durable for long periods or recyclable to use many times.

Alexander Kibler commented: When considering the protein backbone that supports the catalyst, do you think that there are limitations to the scope of products form carbon dioxide reduction? For example, the formation of reduction 
products with strong hydrogen bonding capabilities or low $\mathrm{p} K_{\mathrm{a}}$ value such as formic acid could interact with and destabilise the intramolecular protein interactions or the hydrogen-bond bridged dimeric catalyst.

Charles Machan responded: Certainly one can imagine that if we are limited to strictly biological systems that this approach could potentially be limited by the deleterious effects you describe. The important point about this system is that we are not limited to strictly biologically based peptide sequences; we are free to use nonnatural amino acids which may be robust. We want to develop an understanding of how non-covalent secondary-sphere effects can influence a catalytic process, not necessarily how it would harmonize with naturally occurring peptide sequences.

Vincent Artero opened a discussion of the paper by Osamu Ishitani: Can you comment on the selectivity for CO versus formic acid formation with your photocatalysts? It seems it's not the same as previously described in the literature under electrocatalytic conditions for similar catalysts.

Osamu Ishitani answered: The reaction solution used in this manuscript (DMA- $\mathrm{H}_{2} \mathrm{O} 9: 1 \mathrm{v} / \mathrm{v}$ ) is different to those previously reported (DMA-TEOA $4: 1$ or $5: 1)$. This difference should affect the product distribution in the $\mathrm{CO}_{2}$ reduction. Even in the DMA- $\mathrm{H}_{2} \mathrm{O}$ solution, the photocatalytic system with a $\left[\mathrm{Ru}\left(\mathrm{N}^{\wedge} \mathrm{N}\right)(\mathrm{CO})_{2-}\right.$ $\mathrm{Cl}_{2}$ ] type catalyst gave $\mathrm{HCOOH}$ as a main product. On the other hand, the system with a $\left[\mathrm{Ru}\left(\mathrm{N}^{\wedge} \mathrm{N}^{\wedge} \mathrm{N}\right)\left(\mathrm{Cl}_{2} \text { bpy }\right)(\mathrm{CO})\right]^{+}$type catalyst mainly afforded $\mathrm{CO}$.

Jose Martinez remarked: Can you comment on why you used an ethylene linked bridge versus an ether bridge in your two supramolecular photocatalysts?

Osamu Ishitani answered: Only the synthetic reason. The ethylene chain can be easily used for connecting two same diimine moieties but not for different ones. We recently reported mew methods for connecting different kinds of diimine moieties. . $^{1,2}$

1 Y. Yamazaki, A. Umemoto and O. Ishitani, Inorg. Chem., 2016, 55, 11110-11124.

2 Y. Yamazaki, T. Morimoto and O. Ishitani, Dalton Trans., 2015, 44, 11626-11635.

Haruo Inoue remarked: You showed two types of interesting supramolecular systems. One is forming formic acid and the other prefers CO formation. In near future, I suppose that you would attach them on p-type semiconductors. In this case, are you able to put ionic substituents on the terminal end of the ligand which coordinates to the Ru center to distance them from the surface of semiconductor in order to prevent unexpected back electron transfer?

Osamu Ishitani answered: Suppression of backward electron transfer from the reduced catalyst unit to the semiconductor is one of the most important factors for improving photocatalysis of the hybrids consisting of the supramolecular photocatalyst and semiconductor materials, such as electrodes and particles. Electrostatic repulsion is a potential method, and it is possible to introduce this into our systems. However, since the charges of both the catalyst and photosensitizer units should change during the photocatalytic reactions, exact control of this might be 
difficult. Our strategy described in the paper is that the rigidity of the photosensitizer unit might be applied to keep a longer distance between the catalyst unit and the semiconductor surface. For this purpose, we introduced two components into the photosensitizer unit, i.e., two tridentate ligands and four anchoring groups.

Leif Hammarström commented: You explained the advantages of linking the sensitizer and catalyst. There are some disadvantages. In addition to the synthetic effort, the catalyst could quench the excited state in an unproductive manner, by many different mechanisms. You report lifetimes of excited states at the start, but the reduced catalyst can quench the next excited state by sending the electron back, or by energy transfer. You have an overall quantum yield of $\mathrm{CO}_{2}$ reduction of $5-10 \%$. Could this be one reason why you lose quantum yield?

Osamu Ishitani replied: In many photocatalytic reactions in homogenous solution, the rate limiting steps are the following: (1) light absorption of the photosensitizer and (2) electron transfer from the reduced photosensitizer to the catalyst. In many cases using good catalysts such as $\mathrm{Re}(\mathrm{I})$ and $\mathrm{Ru}(\mathrm{II})$ diimine carbonyl complexes, $\mathrm{CO}_{2}$ reduction after step (2), which contains many processes including the second reduction, is rapid enough. If the step (1) is the slowest, no drastic spectral change of the reaction solution during the photocatalytic reaction is observed. If the step (2) is the slowest, accumulation of the one-electronreduced species (OERS) of the photosensitizer in the reaction solution is observed. The photocatalytic reaction using $\mathrm{Ru}-\mathrm{Ru}(\mathrm{Clbpy})(\mathrm{CO})$ should follow the latter case after $3 \mathrm{~min}$ irradiation when all of the $\mathrm{Ru}(\mathrm{Clbpy})(\mathrm{CO})$ has been converted to $\mathrm{Ru}-\mathrm{Ru}(\mathrm{Clbpy})($ solvent). Since the redox potential of the $\mathrm{Ru}(\mathrm{Clbpy})$ (solvent) catalyst unit is more negative than that of the photosensitizer unit, the electron transfer from the OERS of the photosensitizer unit to the catalyst unit should be slow, and the OERS of the photosensitizer unit accumulates in solution as shown in Fig. 5a of the paper. This situation induces so-called 'inner filter effect' to lower the efficiency of the photocatalytic reaction because the OERS has strong absorption at $480 \mathrm{~nm}$ which is the irradiation wavelength. This should be one of the main reasons for the low quantum yield in the case $\mathrm{Ru}-\mathrm{Ru}(\mathrm{Clbpy})(\mathrm{CO})$. In the case of $\mathrm{Ru}-\mathrm{Ru}(\mathrm{CO})_{2} \mathrm{Cl}_{2}$, this situation does not occur. The intramolecular electron transfer from the OERS of the photosensitizer unit to the catalyst unit should be fast enough and the following processes must also be faster than the light-absorption process by the photosensitizer unit as we did not see any absorption change of the reaction solution during the photocatalytic reaction as shown in Fig. $5 \mathrm{~b}$ of the paper. In such cases, the added electron into the catalytic unit should be 'consumed' via processes such as reaction with $\mathrm{CO}_{2}$ and/or loss of the ligand. Therefore, electron transfer quenching of the excited photosensitizer unit by the OERS of the catalyst unit is not a concern. Good examples of supramolecular photocatalysts can be found in the literature. ${ }^{1,2}$

The quantum yields of the $\mathrm{CO}_{2}$ reduction were $45-46 \%$.

Another factor contributing to a low quantum yield of the $\mathrm{CO}_{2}$ reduction is rapid backward electron transfer after the initial photochemical electron transfer between the excited photosensitizer and the reductant. In this process, both while in the solvent cage and after the solvated ion pair is separated, the quantum yield of $\mathrm{CO}_{2}$ reduction is lowered. Even in the cases using the supramolecular photocatalysts, this is a serious problem. We analyzed this process in detail. ${ }^{2}$ 
We have not investigated the efficiency of formation of the OERS of the Ru$\mathrm{Ru}(\mathrm{CO})_{2} \mathrm{Cl}_{2}$ photosensitizer unit. This might be lower than those using the supramolecular photocatalysts reported in the references 1 and 2. I also have to point out, that the quenching ratio of the excited photosensitizer units by BIH did not proceed quantitatively $(\sim 80 \%)$. This also lowered the quantum yield of the photocatalytic reactions.

1 Y. Tamaki, D. Imori, T. Morimoto, K. Koike and O. Ishitani, Dalton Trans., 2016, 45, 1466814677.

2 Y. Tamaki, K. Koike, T. Morimoto and O. Ishitani, J. Catal., 2013, 304, 22-28.

Leif Hammarström asked: There are many intermediate states of the catalyst that could potentially quench. A strategy to avoid this is to have a weak coupling link to the catalyst and a strong one to the semiconductor, so the initial excited state reaction is always the interfacial charge transfer.

Osamu Ishitani replied: I agree that interfacial electron transfer from the semiconductor to the photosensitizer is one of the key steps in the hybrid systems. However, we do not have detailed information about this. There are many factors which we have to clarify, such as distances not only between the semiconductor surface and the photosensitizer unit but also between the semiconductor and the catalyst unit. How does the charge variation of the photosensitizer and catalyst units affect relative positions of these three components during the photocatalytic reaction? I believe that basic research using various techniques is needed for clarifying and improving this important and interesting process.

Michael Wasielewski asked: Following up on Professor Hammerström's comment, when the photosensitizer transfers one electron to the catalyst, the catalyst is then paramagnetic, so that spin exchange quenching of the adjacent photosensitizer, which must be photoexcited again to carry out the second reduction in the catalytic cycle, can decrease catalyst performance. Do you see any evidence of such quenching in your system?

Osamu Ishitani responded: The quantum yields of $\mathrm{CO}_{2}$ reduction using the supramolecular photocatalysts which we have constructed were mostly higher than those of the corresponding mixed systems using mononuclear complexes. I do not think that spin exchange quenching is a serious problem in our systems. As I already mentioned, the added electron in the catalytic unit should be 'consumed' via the following processes such as reaction with $\mathrm{CO}_{2}$ and/or loss of the ligand.

Etsuko Fujita addressed Osamu Ishitani and Yusuke Tamaki: Table 2 in your paper contains reduction potentials of complexes used in your work. When we compare the first reduction potentials of $\mathrm{Ru}(\mathrm{dmb})(\mathrm{CO})_{2} \mathrm{Cl}_{2}(-1.65 \mathrm{~V})$ and $\mathrm{Ru}-$ $\mathrm{Ru}(\mathrm{CO}))_{2} \mathrm{Cl}_{2}(-1.53 \mathrm{~V})$, you observed an over $100 \mathrm{mV}$ shift. However, in the case of $\mathrm{Ru}-\mathrm{Ru}(\mathrm{Clbpy})(\mathrm{CO})$, there appears to be no such shift. In the former case, the shift is favourable for a larger driving force for photoinduced electron transfer from the 
sensitizer to the catalyst. How did you guess the structure to construct the supramolecule with such an amazing result? Is the shift due to the ether bridge?

Osamu Ishitani responded: We reported only one supramolecular photocatalyst where the $\mathrm{Ru}$ photosensitizer unit and the Re catalyst unit were connected to each other with an ether chain, i.e., $-\mathrm{CH}_{2} \mathrm{OCH}_{2}-$. In this case, the shifts of redox potential of the photosensitizer unit ( $\mathrm{PS} / \mathrm{PS}^{-\bullet}$ ) and the catalyst unit (CAT/CAT ${ }^{-\bullet}$ ) from the corresponding mononuclear complexes with 4,4'-dmb were $10 \mathrm{mV}$ and $50 \mathrm{mV}$, respectively. ${ }^{1}$ In the case of $\mathrm{Ru}-\mathrm{Ru}(\mathrm{CO})_{2} \mathrm{Cl}_{2}$, on the other hand, the structure of the ether chain was unsymmetrical, i.e., (PS)- $\mathrm{OCH}_{2}-(\mathrm{CAT})$, where the electronic effect of the oxygen atom should be different between the PS and CAT units as described below. Fig. 3a shows the CVs of both $\mathrm{Ru}-\mathrm{Ru}(\mathrm{CO})_{2} \mathrm{Cl}_{2}$ and the model compounds. The irreversible wave with a peak at $-1.53 \mathrm{~V}$ in the case of $\mathrm{Ru}-$ $\mathrm{Ru}(\mathrm{CO})_{2} \mathrm{Cl}_{2}$ can be attributed to a one-electron reduction of the CAT unit. Because of this irreversibility, we cannot determine the exact positive shifts of the redox potential of $\mathrm{Ru}-\mathrm{Ru}(\mathrm{CO})_{2} \mathrm{Cl}_{2}$ from those of the model complexes, but it should be about $100 \mathrm{mV}$. We do not know the reason for the $\sim 50 \mathrm{mV}$ difference in the reduction potential shifts of the CAT unit compared to the $\mathrm{Ru}-\mathrm{Re}$ supramolecular photocatalyst. As the first reduction wave of the model mononuclear complex of PS was reversible, an irreversible reaction at the second reduction attributed to one-electron reduction of the PS unit of $\mathrm{Ru}-\mathrm{Ru}(\mathrm{CO})_{2} \mathrm{Cl}_{2}$ should proceed. This potential of $\mathrm{Ru}-\mathrm{Ru}(\mathrm{CO})_{2} \mathrm{Cl}_{2}$ was slightly more negative than that of the model complex $[(\mathrm{mbip}) \mathrm{Ru}(\mathrm{mtpy})]^{2+}$. This is reasonable because the oxygen atom directly attached to the aromatic ligand of the PS unit should give weak electron-donating effect. Fig. 3 shows CVs of Ru-Ru(Clbpy)(CO) where the large shifts of redox potentials were not observed because there should not be strong electric
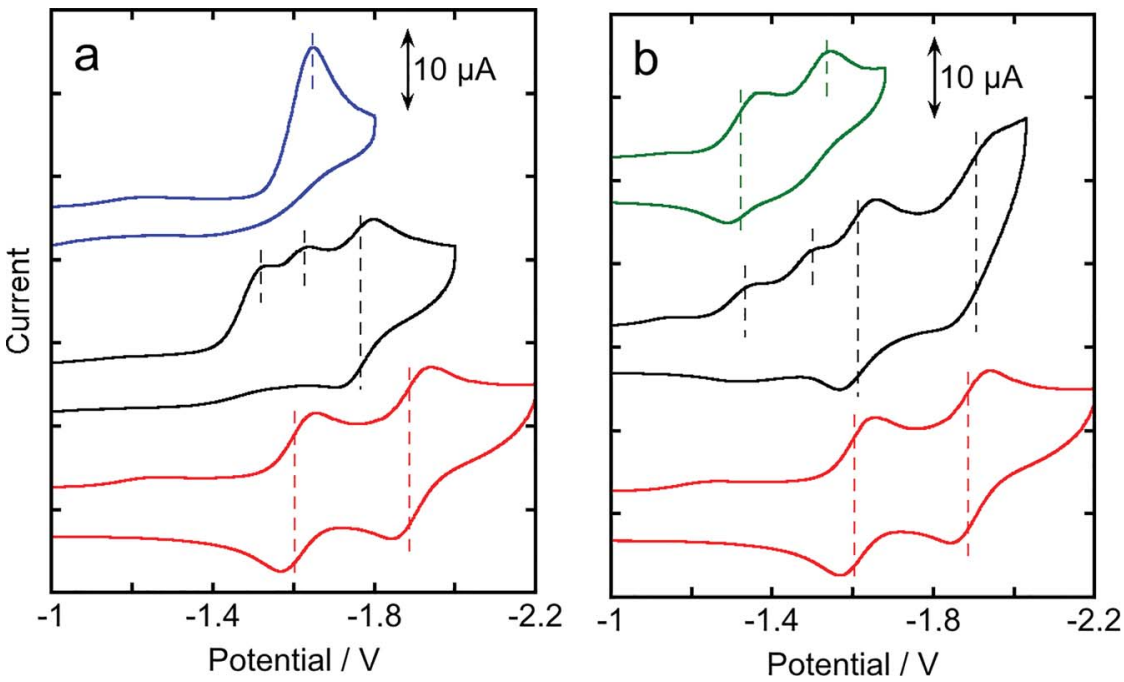

Fig. 3 Cyclic voltammograms of (a) $\mathrm{Ru}-\mathrm{Ru}(\mathrm{CO})_{2} \mathrm{Cl}_{2}$ (black) and the model compounds (blue: $\mathrm{Ru}(\mathrm{dmb})(\mathrm{CO})_{2} \mathrm{Cl}_{2}$, red: $\left.[(\mathrm{mbip}) \mathrm{Ru}(\mathrm{mtpy})]^{2+}\right)$, and (b) $\mathrm{Ru}-\mathrm{Ru}(\mathrm{Clbpy})(\mathrm{CO})$ (black) and the model compounds (green: $\mathrm{Ru}(\mathrm{dmb})(\mathrm{CO})_{2} \mathrm{Cl}_{2}$, red: $\left.[(\operatorname{mbip}) \mathrm{Ru}(\mathrm{mtpy})]^{2+}\right)$. 
interaction between the PS and CAT units owing to the ethylene chain in the bridging ligand.

1 E. Kato, H. Takeda, K. Koike, K. Ohkubo and O. Ishitani, Chem. Sci., 2015, 6, 3003-3012.

Haruo Inoue opened discussion of the paper by Ho-Jin Son: Congratulations on your finding that the conduction band potential is controlled by choosing the solvent composition. May I ask about the mechanism of the $\mathrm{CO}_{2}$ reduction on $\mathrm{TiO}_{2}$ ? Does this proceed through simultaneous two-electron transfer just like the electrochemical reduction, or through stepwise electron transfer processes as observed in photochemical reaction in homogeneous solution? If we have information on how frequently an electron is injected into the conduction band of $\mathrm{TiO}_{2}$, that is, how many dye molecules are adsorbed on the $\mathrm{TiO}_{2}$ surface (surface coverage), how frequently they absorb photons, and how many Re complexes are sharing the injected electrons, we could discuss this point.

Ho-Jin Son replied: We now think that the catalytic $\mathrm{CO}_{2}$ reduction will follow stepwise electron transfer from $\mathrm{TiO}_{2}$ to the anchored $\mathrm{Re}(\mathrm{I})$ because the catalytic $\mathrm{CO}_{2}$ reduction in our hybrid system involves multi-step, complex chemical processes (the slow $\mathrm{CO}_{2}$ coordination to Re center, the liberation of $\mathrm{Cl}^{-}$, and coordination of a solvent molecule) as suggested in many previous results. Fortunately, the reported time scale $(\sim 400 \mathrm{~ms})$ from $\mathrm{TiO}_{2}(\mathrm{e}-)$ to a $\operatorname{Re}(\mathrm{I})$ catalyst $^{1}$ evidences such stepwise electron transfer processes. We now believe that the real time scale is similar to the reported value $(\sim 400 \mathrm{~ms})$ although this value is not based on our photocatalytic conditions $\left(\mathrm{CO}_{2}\right.$-saturated DMF solution with dye) but on the limited conditions used for their time-resolved spectroscopy experiments. The $\mathrm{CO}_{2}$ reduction processes can be considered to be slower than the electron-injection processes (from the anchored dye to $\mathrm{TiO}_{2}$ ), a situation that leads to a mismatch between the electron injection $(\sim \mathrm{ps})$ and the chemical processes $(\sim \mathrm{ms})$. As the consequence, the injected $\mathrm{TiO}_{2}(\mathrm{e}-)$ might be increasingly accumulated in $\mathrm{TiO}_{2}$. This means that the $\mathrm{TiO}_{2}(\mathrm{e}-)$ could await the generation of an electron-accepting intermediate during or after the chemical processes with the sufficient lifetime of injected electrons in $\mathrm{TiO}_{2}$.

1 J. Durrant, E. Reisner et al., Chem. Eur. J., 2015, 21, 3746.

Haruo Inoue asked: What is the time scale of the reduction process?

Ho-Jin Son responded: We didn't measure the exact rate for catalytic $\mathrm{CO}_{2}$ reduction. However, a study by Durrant and Reisner et al. reports the time scale from $\mathrm{TiO}_{2}(\mathrm{e}-)$ to the $\mathrm{Re}(\mathrm{I})$ catalyst is approximately $\sim 400 \mathrm{~ms} .{ }^{1}$ Although this value is not based on our photocatalytic conditions $\left(\mathrm{CO}_{2}\right.$-saturated DMF solution with dye) but on the limited conditions used for their time-resolved spectroscopy experiments, we believe that the real time scale is similar to this measured value ( $\sim 400 \mathrm{~ms}$ ) because the catalytic $\mathrm{CO}_{2}$ reduction in our hybrid system also follows the monomeric mechanism involving the complex chemical process involving slow $\mathrm{CO}_{2}$ coordination as suggested in many previous results.

1 J. Durrant, E. Reisner et al., Chem. Eur. J., 2015, 21, 3746. 
Christopher Windle asked: In your system you irradiate with wavelengths $>400 \mathrm{~nm}$ and will therefore excite some of the Re. Have you done an experiment in the absence of dye and can you comment on the TON of CO in this case?

Ho-Jin Son replied: Yes, we did. As you suggested, there is a overlap between absorption of Re and $>400 \mathrm{~nm}$ of LED light source, but the intensity of the LED used is very low at $\sim 400 \mathrm{~nm} .{ }^{1}$ Indeed, the $\mathrm{TiO}_{2} /$ Re sample showed a TON of only $\sim 30$ with a tendency to level off relatively early.

1 J.-S. Lee, D.-I. Won, W.-J. Jung, H.-J. Son, C. Pac and S. O. Kang, Angew. Chem. Int. Ed., 2017, 56, 976-980.

Peter Summers asked: In Fig. 2 of your paper, the systems seems relatively stable up to $30 \mathrm{~h}$ of irradiation, have you tested them for any longer?

Ho-Jin Son answered: The activity is continued up to $\sim 50 \mathrm{~h}$ with slight levelling off behavior. We now think the photocatalytic process after $30 \mathrm{~h}$ can retain visiblelight harvesting properties of the photomodified organic sources on the $\mathrm{TiO}_{2}$ surface (remaining after the photobleaching). In our unpublished work, we observed visible-light absorption peaks remaining unchanged after significant photobleaching-albeit, to a lesser degree than observed with original absorption of the dye before irradiation.

Peter Summers commented: Do you know what is limiting CO production in some experiments? Is it dye/catalyst decomposition or dissociation from the $\mathrm{TiO}_{2}$ surface? Have you checked the solvent and $\mathrm{TiO}_{2}$ after the reaction?

Ho-Jin Son replied: In our hybrid system, the main deactivation process is caused by photobleaching of dye used relative to catalyst. After $\sim 30 \mathrm{~h}$, the initial orange color $\mathrm{OrgD} / \mathrm{TiO}_{2} / \mathrm{Re}(\mathrm{I})$ particles have become pale yellow. The related DRS data also showed a significant decrease of absorption peaks of dyes used. However, the activity is continued up to $\sim 50 \mathrm{~h}$ with a slight levelling off behavior as mentioned previously.

Akihiko Kudo asked: Have you measured an action spectrum?

Ho-Jin Son responded: No, we are planning to set up a measurement tool for obtaining action spectrum.

Akihiko Kudo asked: What are the crystal phases of your samples? If the samples contain rutile, they respond to $400 \mathrm{~nm}$ of the irradiation. So, control experiments with only $\mathrm{TiO}_{2}$ samples are necessary. The action spectra are also informative.

Ho-Jin Son responded: We used Hombikat UV-100 $\mathrm{TiO}_{2}(\sim 99 \%$ anatase) purchased from Huntsman P\&A GmbH. Moreover, we now think the possibility of $\mathrm{TiO}_{2}$ excitation under $>400 \mathrm{~nm}$ (LED) is very low because the intensity of LED used is very low at $\sim 400 \mathrm{~nm}$, as I answered to the question from Christopher Windle. 
Also, in our experiment, the control experiment $\left(\right.$ dye $\left./ \mathrm{TiO}_{2}\right)$ did not show any CO conversion efficiency (see the ESI in reference 1). ${ }^{1}$

1 H.-J. Son, C. Pac and S. O. Kang et al., J. Am. Chem. Soc., 2015, 137, 13679

Leif Hammarström opened a general discussion of the papers by Osamu Ishitani and Ho-Jin Son: You present two principally different photocathodes: Osamu Ishitani with $\mathrm{NiO}$ as the ultimate donor, and Ho-Jin Son with the dye as the ultimate donor. If you couple these to anodes for water oxidation, this has to be done very differently. I would imagine that Osamu Ishitani would wire NiO to the photoanode material $\left(\right.$ e.g. $\left.\mathrm{TiO}_{2}\right)$ as has been done, while Ho-Jin Son would have a wireless system and instead a molecular redox shuttle between anode and cathode dyes, similar to the particulate systems of, for example, Kazunari Domen and coworkers. Can you comment on pros and cons of the approaches? What material would Ho-Jin Son use for his anode?

Akihiko Kudo answered: All possible systems, not focusing on a particular system, should be examined because each approach has merits and demerits.

Ho-Jin Son responded: We are now trying to build an efficient Z-scheme system using water as electron source. However, there are lots of factor to considered. Honestly, we do not have enough information yet. Energy levels would be significantly affected by the solvent media used. For example, the conduction band of $\mathrm{TiO}_{2}$ semiconductor will be dramatically lowered by $\sim-0.5 \mathrm{~V} v$ s. SCE, resulting in a bad electron transfer rate from $\mathrm{TiO}_{2}$ to the anchored electrocatalyst (i.e., $-1.34 \mathrm{~V} v$ s. SCE for Re(I) catalyst). Therefore, an appropriate treatment must be needed for use in water media. In addition, the energy level alignment (for the regeneration of the dye radical cation) between the excited state reduction potential of the dye and the oxidation potential of the redox shuttle would be essential in the fabrication of a Z-scheme photocatalytic system using Dye/ $\mathrm{TiO}_{2} /$ Cat. as an anode for $\mathrm{CO}_{2}$ reduction.

Sang Ook Kang asked: Regarding the isomeric forms of the supramolecular photocatalyst anchored on to the semiconductor surface, how do you control the conformation with respect to the close proximity to the semiconductor surface, and determine that one isomer is better than the other for charge recombination?

Osamu Ishitani responded: This is the purpose of our research reported in this paper. Our previous reports ${ }^{1-5}$ describe supramolecular photocatalysts consisting of the $\left[\mathrm{Ru}\left(\mathrm{N}^{\wedge} \mathrm{N}\right)_{3}\right]^{2+}$ type photosensitizer where the three $\mathrm{N}^{\wedge} \mathrm{N}$ ligands have different substituents, i.e., bridging ethylene chain, anchoring groups, and just methyl groups. Therefore, we had to use various isomers of the supramolecular photocatalyst to construct the hybrids because isolation of each isomer is very difficult. This should cause different distances between the catalyst unit and the surface of the semiconductor. We described this situation in the introduction section of our paper. Our proposal in this paper is to introduce two tridentate ligands into the photosensitizer unit of the supramolecular photocatalyst to simplify this problem. This should make the distance between the catalyst and 
the semiconductor more rigid and longer, because of the trans positions between the two ligands with the bridging ethylene chain or anchoring groups.

1 H. Kumagai, K. Maeda, N. Kaeffer, V. Artero, M. Higashi, R. Abe and O. Ishitani, J. Am. Chem. Soc., 2016, 138, 14152-14158.

2 K. Wada, M. Eguchi, O. Ishitani and K. Maeda, ChemSusChem, 2017, 10, 287

3 A. Nakada, T. Nakashima, K. Sekizawa, K. Maeda and O. Ishitani, Chem. Sci., 2016, 7, 43644371.

4 R. Kuriki, H. Matsunaga, T. Nakashima, K. Wada, A. Yamakata, O. Ishitani, and K. Maeda, J. Am. Chem. Soc., 2016, 138, 5159-5170.

5 K. Muraoka, H. Kumagai, M. Eguchi, O. Ishitani and K. Maeda, Chem. Commun., 2016, 52, 7886-7889.

Daniel Nocera asked: What do you see as advantages of molecular $\mathrm{CO}_{2}$ reduction as compared to heterogeneous reduction methods? I'll give you one that I think is important. In heterogeneous systems, it has been shown that many heterogeneous catalysts aren't selective for $\mathrm{CO}_{2}$ but rather they suppress hydrogen, thus allowing CO reduction to occur (e.g., nanoparticles that they make from oxides exhibit a high density of strained boundaries and for some odd reason, strain results in hydrogen suppression). Molecular systems may target $\mathrm{CO}_{2}$ over $\mathrm{H}_{2}$ with design and they are more tunable. A second advantage is that molecular catalysts can be dispersed on solid supports and thus have, in principle, a higher activity per catalyst center. Do you see others?

Osamu Ishitani responded: The selectivity of $\mathrm{CO}_{2}$ reduction is not so simple because it is strongly dependent on various conditions. For example, the photocatalysis of most of molecular photocatalysts has been tested in organic solutions, or containing a small portion of water in very limited number of cases. In the future, we will have to use water as a reductant. This drastic change of solvent should affect the selectivity of $\mathrm{CO}_{2}$ reduction $v s . \mathrm{H}_{2}$ evolution. If you use an aqueous solution, what is the $\mathrm{pH}$ ? We can also discuss this point in the cases using semiconductor photocatalysts. Recently, we have been testing the photocatalysis of both the supramolecular photocatalysts and their hybrids with semiconductors in aqueous solutions. ${ }^{1-4}$ The most important points should be how the hydride species is produced and how it can react with $\mathrm{CO}_{2}$ and $\mathrm{H}^{+}$in both molecular catalysts and heterogeneous catalysts. We do not have enough information about these points.

Anyway, in answer to the question from Professor Nocera: we might be able to separate the oxidation and reduction sites, i.e., places for $\mathrm{CO}_{2}$ reduction and water oxidation, if two kinds of molecular catalysts can be selectively adsorbed on the exact positions. We might use different anchor groups for this purpose, and we have to develop new adsorption methods of metal complexes on the various type of surfaces.

1 A. Nakada, T. Nakashima, K. Sekizawa, K. Maeda and O. Ishitani, Chem. Sci., 2016, 7, 43644371

2 A. Nakada, K. Koike, K. Maeda and O. Ishitani, Green Chem., 2016, 18, 139-143.

3 A. Nakada, K. Koike, T. Nakashima, T. Morimoto and O. Ishitani, Inorg. Chem., 2015, 54, 1800-1807.

4 R. Kuriki, M. Yamamoto, K. Higuchi, Y. Yamamoto, M. Akatsuka, D. Lu, S. Yagi, T. Yoshida, O. Ishitani and K. Maeda, Angew. Chem. Int. Ed., 2017, in press. 